\title{
Some Puzzles and Unresolved Issues about Quantum Entanglement
}

\section{John Earman}

Department of History and Philosophy of Science, University of Pittsburgh, Pittsburgh, PA 15260, USA

e-mail: jearman@pitt.edu

Abstract Schrödinger (1935) averred that entanglement is the characteristic trait of quantum mechanics. The first part of this paper is simultaneously an exploration of Schrödinger's claim and an investigation into the distinction between mere entanglement and genuine quantum entanglement. The typical discussion of these matters in the philosophical literature neglects the structure of the algebra of observables, implicitly assuming a tensor product structure of the simple Type I factor algebras used in ordinary QM. This limitation is overcome by adopting the algebraic approach to quantum physics, which allows a uniform treatment of ordinary QM, relativistic QFT, and quantum statistical mechanics. The algebraic apparatus helps to distinguish several different criteria of quantum entanglement and to prove results about the relation of quantum entanglement to two additional ways of characterizing the classical vs. quantum divide, viz. abelian vs. non-abelian algebras of observables, and the ability vs. inability to interrogate the system without disturbing it. Schrödinger's claim is reassessed in the light of this discussion. The second part of the paper deals with the relativity-to-ambiguity threat: the entanglement of a state on a system algebra is entanglement of the state relative to a decomposition of the system algebra into subsystem algebras; a state may be entangled with respect to one decomposition but not another; hence, unless there is some principled way to choose a decomposition, entanglement is a radically ambiguous notion. The problem is illustrated in terms a Realist vs. Pragmatist debate, the former claiming that the decomposition must correspond to real as opposed to virtual subsystems, while the latter claims that the real vs. virtual distinction is bogus and that practical considerations can steer the choice of decomposition. This debate is applied to the fraught problem of measuring entanglement for indistinguishable particles. The paper ends with some (intentionally inflammatory) remarks about claims in the philosophical literature that entanglement undermines the separability or independence of subsystems while promoting holism.

\section{Introduction}


For Schrödinger, one of the founders of quantum mechanics (QM), entanglement is the characteristic feature of QM that separates it from classical mechanics. The opening sentences of Schrödinger (1935) read:

When two systems, of which we know the states by their respective representatives, enter into temporary physical interaction due to known forces between them, and when after a time of mutual influence the systems separate again, then they can no longer be described in the same way as before, viz. by endowing each of them with a representative of its own. I would not call that one but rather the characteristic trait of quantum mechanics, the one that enforces its entire departure from classical lines of thought. By the interaction the two representatives (or $\psi$-functions) have become entangled. (p. 555; italics in original)

Obvious counterclaims would be that the characteristic trait of quantum systems lies in the non-abelian nature of the observables, or in the fact that quantum systems cannot be measured without disturbance while classical systems can. It turns out that Schrödinger's claim and the counterclaims are closely related, at least on one understanding of what quantum entanglement is. That is what this paper is about: it is not about the moaning over spooky action-at-a-distance that accompanies (some forms of) entanglement but about how to characterize quantum entanglement and about how this entanglement marks what is distinctive of the quantum realm. ${ }^{1}$ The need for such a treatment arises from the fact that, despite the many hundreds (thousands?) of papers on entanglement, there are still puzzles and unresolved issues. Mathematical physicists are well aware of these issues but curiously most philosophers of science seem oblivious, even though some of the issues have interesting philosophical content and even though philosophers are well equipped to contribute. The purpose of this paper is to lay out the issues in a way that will engage philosophers who are interested in the foundations of quantum physics.

The paper is organized as follows. Section 2 introduces the algebraic approach to quantum physics which, I claim, promotes clear-headed thinking about entanglement. In the version of the approach used here a system is characterized in terms of a von Neumann algebra of observables and a state on the algebra. Entanglement of this state is entanglement relative to a choice 
of subalgebras of observables corresponding to subsystems. In Sections 3-7 it is taken for granted that the choice of subsystem algebras has been made, and the focus is on finding criteria for quantum entanglement over these subalgebras. The search is guided by two ideas: first, that the correlations contained in genuine quantum entanglement should not be producible by a classical mechanism; and, second, that the analysis of quantum entanglement should capture what is characteristic about the entanglement of states over non-abelian subalgebras of quantum observables as opposed to entanglement over the abelian subalgebras of classical observables. These ideas lead to several distinct criteria for quantum entanglement that form a strict hierarchy of increasing strength. As with all explications, formal criteria never square completely with intuitions, and some of the misfits are detailed in Sections 6 and 7 . Results about the ubiquity of quantum entanglement are detailed in Section 8. Section 9 then revisits Schrödinger's claims about entanglement to give both a partly sympathetic and a partly non-sympathetic assessment in light of what we have learned about entanglement over the intervening three quarters of a century. Section 10 starts with a truism - that entanglement means entanglement of a state on a system algebra with respect to a decomposition of the system algebra into subsystem algebras - and an obvious fact - that the very same system state may be unentangled with respect to one decomposition but entangled with respect to another. Unless there are limitations on the choice of decompositions, this relativity of entanglement results in a hopeless ambiguity of entanglement. A Realist would respond that real entanglement concerns entanglement over subalgebras corresponding to real as opposed to virtual subsystems, and that where there is no principled way to draw the distinction questions of entanglement become moot. The Pragmatist responds that there is no valid metaphysical basis for drawing the distinction between real and virtual systems; but practical factors, such as experimental accessibility, can make it reasonable to work with one decomposition rather than another. Since these practical factors vary with context, so does entanglement. The Realist vs. Pragmatist debate is illustrated in terms of concrete examples. Section 11 introduces the fraught issue of how to talk about entanglement for indistinguishable particles. The physics literature contains proposals to measure the depth of entanglement for vector states for fermionic and bosonic systems. These are formal requirements stated as conditions on Hilbert space vectors. But since nothing is said about the decomposition of the system algebra into subsystem algebra, it is pertinent to ask "What are they talking about when they conclude from the 
satisfaction (respectively, failure) of the formal criteria that entanglement holds (respectively, fails)?" The Realist vs. Pragmatist debate is brought to bear on this question. Section 12 contains conclusions, along with a closing rant about attempts in the philosophical literature to relate entanglement to non-separability of physical systems and to holism.

\section{The Apparatus}

The present discussion of entanglement will employ the algebraic approach to quantum physics, one advantage being that it provides a unified way to talk about entanglement in ordinary non-relativistic quantum mechanics (QM), relativistic quantum field theory (QFT), and quantum statistical mechanics. Additionally, starting with algebras helps to underscore a blindingly obvious point that, despite its crucial nature, seems to elude many commentators; namely, while the typical discussion of entanglement focuses on states, the algebras of observables come first; for the entanglement of a state must be understood as relative to a choice of (sub)system algebras, and the very same state that is entangled with respect to one choice may be unentangled with respect to another. This observation will be the starting point for Section 10, but before reaching that juncture much initial spade work needs to be done. The present Section provides a brutally brief introduction to the algebraic the apparatus. Readers interested in more in depth treatments can consult any number of good texts (e.g. Haag 1992, Horuzhy 1990, and Bratelli and Robinson 1987).

A von Neumann algebra $\mathfrak{N}$ is a *-algebra of bounded operators acting on a Hilbert space $\mathcal{H} .^{2}$ It is required that $\mathfrak{N}$ be closed in the weak operator topology or, equivalently (by von Neumann's double commutant theorem) $\mathfrak{N}^{\prime \prime}:=\left(\mathfrak{N}^{\prime}\right)^{\prime}=\mathfrak{N}$ where "' denotes the commutant (consisting of all the bounded operators that commute with all the element of $\mathfrak{N})$. The most familiar example comes from ordinary QM (sans superselection rules) where the algebra of observables is (isomorphic to) the Type I factor algebra $\mathfrak{B}(\mathcal{H})$, the algebra of all bounded operators acting on $\mathcal{H} .{ }^{3}$ In the algebraic approach to relativistic QFT algebras of observables are associated with open bounded regions of Minkowski spacetime, and these algebras are typically Type III. To give but one example of how exotic these latter algebras are, at least to someone raised on ordinary QM, I mention that they do not contain any finite dimensional projection operators. 
A state $\omega$ on an algebra $\mathfrak{N}$ is an expectation value functional, i.e. a normed $(\omega(I)=1)$ positive linear functional $\omega: \mathfrak{N} \rightarrow \mathbb{C}$. A state is said to be pure if it cannot be written as a non-trivial convex combination $\omega=$ $\lambda_{1} \varphi_{1}+\lambda_{2} \varphi_{2}, 0<\lambda_{1}, \lambda_{2}<1, \lambda_{1}+\lambda_{2}=1$, where $\varphi_{1}$ and $\varphi_{2}$ are distinct states on $\mathfrak{N}$; otherwise $\omega$ is said to be mixed. A vector state $\omega$ is a state where there is a $|\psi\rangle \in \mathcal{H}$ such that $\omega(A)=\langle\psi|A| \psi\rangle$ for all $A \in \mathfrak{N}$. For $\mathfrak{N}=\mathfrak{B}(\mathcal{H})$ the vector states and the pure states coincide; but the coincidence is broken for more general algebras, e.g. for Type I non-factors and for Type III algebras (where all vector states are mixed). A state $\omega$ on $\mathfrak{N}$ is normal if and only if there is a normed trace class operator ("density matrix") $\varrho$ such that $\omega(A)=\operatorname{Tr}(A \varrho)$ for all $A \in \mathfrak{N}$. Vector states are, or course, normal. An element $E \in \mathfrak{N}$ is a projection if it is self-adjoint and idempotent $\left(E^{2}=E\right)$. The projections of a system algebra $\mathfrak{N}$ are supposed to correspond to Yes-No questions that can be asked about the system, and if $\omega$ is a normal state with corresponding density matrix $\varrho$ then $\omega(E)=\operatorname{Tr}(E \varrho)$ is the probability of a Yes outcome for a measurement of $E$ made in state $\omega$.

The projections $\mathcal{P}(\mathfrak{N})$ of a von Neumann algebra $\mathfrak{N}$ have a natural structure as a lattice. Quantum probability theory can be thought of as the study of probability measures on these non-abelian, orthocomplemented projection lattices. A normal state on $\mathfrak{N}$ induces a countably additive probability measure on $\mathcal{P}(\mathfrak{N})$. By the generalized Gleason theorem, a countably additive measure on $\mathcal{P}(\mathfrak{N})$ has (under mild restrictions on $\mathfrak{N}$ ) a unique extension to a normal state on $\mathfrak{N}{ }^{4}$ It follows that for a countably additive measure on $\mathcal{P}(\mathfrak{N})$ the probabilities must conform to the "Born rule."

In practice physicists consider only normal states, their implicit assumption being that non-normal states are not physically realizable. I know of no convincing argument for this assumption, and it certainly deserves more scrutiny than it has received in the literature; but I will not belabor the point here, and will follow the standard practice of focusing on normal states. But I will note from time to time that issues about entanglement take on a different complexion if non-normal states are admitted.

\section{What is Entanglement?}

In the version of the algebraic approach used here a physical system is characterized by a von Neumann algebra $\mathfrak{N}$, and subsystems of the system are characterized by von Neumann subalgebras $\mathfrak{N}_{1}, \mathfrak{N}_{2}, \ldots .{ }^{5}$ In the present Section a "bottom up" version of this approach is used: start with algebras 
$\mathfrak{N}_{1}$ and $\mathfrak{N}_{2}$ thought of as giving the observable algebras of two subsystems, and define the system algebra to be $\mathfrak{N}_{1} \vee \mathfrak{N}_{2}$, the smallest von Neumann algebra to be generated by $\mathfrak{N}_{1}$ and $\mathfrak{N}_{2}$. One major goal is to specify criteria for a state $\omega$ on $\mathfrak{N}_{1} \vee \mathfrak{N}_{2}$ to be quantum entangled over $\mathfrak{N}_{1}$ and $\mathfrak{N}_{2}$. One implicit assumption (inherited from Schrödinger) of this quest is that quantum entanglement, properly understood, will reveal features that distinguish quantum systems from classical systems. Another assumption that seems to be ingrained in the philosophical literature is that quantum entanglement somehow compromises the separability/independence of subsystems. I think this is a mistake, and I will have more to say about it in Section 12. But at the start I will assume that talk about entanglement of subsystems makes sense only if these subsystems enjoy some minimal degree of separability/independence; and, arguably, the minimal degree would include mutual commutativity of subsystem algebras so that the subsystem observables can be simultaneously measured. In ordinary QM the mutual commutativity of the subsystem algebras corresponding to distinguishable particles is universally if silently imposed. ${ }^{6}$ In the algebraic approach to relativistic QFT, algebras of observables $\mathfrak{N}(\mathcal{O})$ are associated with open bounded regions $\mathcal{O}$ of Minkowski spacetime, and the requirement that $\left[\mathfrak{N}\left(\mathcal{O}_{1}\right), \mathfrak{N}\left(\mathcal{O}_{2}\right)\right]=0$ when $\mathcal{O}_{1}$ and $\mathcal{O}_{2}$ are relatively spacelike is known as micro-causality (aka Einstein causality or Einstein locality).

With this in mind, we meet the basic notion of entanglement in the following:

Def. 1. Let $\mathfrak{N}_{1}$ and $\mathfrak{N}_{2}$ be mutually commuting von Neumann algebras acting on a common Hilbert space.

(i) A state $\omega$ on $\mathfrak{N}_{1} \vee \mathfrak{N}_{2}$ is a product state with respect to $\mathfrak{N}_{1}$ and $\mathfrak{N}_{2}$ iff $\omega(A B)=\omega(B A)=\omega(A) \omega(B)$ for all $A \in \mathfrak{N}_{1}$ and $B \in \mathfrak{N}_{2} \cdot{ }^{7}$

(ii) A state $\omega$ on $\mathfrak{N}_{1} \vee \mathfrak{N}_{2}$ is entangled (E0) with respect to $\mathfrak{N}_{1}$ and $\mathfrak{N}_{2}$ iff it is not a product state.

The connection between non-product states and the existence of correlations in the familiar probabilistic sense can be seen from the application to projection operators. If $C \in \mathfrak{N}_{1} \vee \mathfrak{N}_{2}$ is a projector and $\omega$ is a state on $\mathfrak{N}_{1} \vee \mathfrak{N}_{2}$ then $\omega(C)$ is the probability $\operatorname{Pr}_{\omega}(C)$ in state $\omega$ of a Yes outcome of a measurement of $C$. If $E \in \mathfrak{N}_{1}$ and $F \in \mathfrak{N}_{2}$ are projectors, $\omega$ 's being a product state on over $\mathfrak{N}_{1}$ and $\mathfrak{N}_{2}$ implies that $\operatorname{Pr}_{\omega}(E F)=\operatorname{Pr}_{\omega}(E) \operatorname{Pr}_{\omega}(F)$, the 
usual criterion for no correlation between $E$ and $F$; and since von Neumann algebras are generated by their projections, it follows for a normal state $\omega$ on $\mathfrak{N}_{1} \vee \mathfrak{N}_{2}$ that $\operatorname{Pr}_{\omega}(E F)=\operatorname{Pr}_{\omega}(E) \operatorname{Pr}_{\omega}(F)$ for all projectors $E \in \mathfrak{N}_{1}$ and $F \in \mathfrak{N}_{2}$ if and only if $\omega$ is a product state over $\mathfrak{N}_{1}$ and $\mathfrak{N}_{2}$. Or stated in terms of entanglement in the sense of Def. 1, a state on $\mathfrak{N}_{1} \vee \mathfrak{N}_{2}$ is entangled (E0) with respect to $\mathfrak{N}_{1}$ and $\mathfrak{N}_{2}$ iff it entails a non-zero correlation between some of the subsystem observables.

If this is all entanglement amounts to, then (the reader may well wonder) what's all the shouting about? The short answer is that quantum entanglement involves much more than is captured in Def. 1 . The more will unfold in the coming sections. But before moving on it is worth pausing to note that the algebraic apparatus already points to some differences between the quantum and the classical. In particular, if both of the subsystem algebras $\mathfrak{N}_{1}$ and $\mathfrak{N}_{2}$ are classical (= abelian) then every pure state - normal or noton $\mathfrak{N}_{1} \vee \mathfrak{N}_{2}$ is a product state, so entanglement (E0) is possible for classical systems only for mixed states. ${ }^{8}$ For quantum (= non-abelian) subsystem algebras, entanglement (E0) is possible for pure as well as mixed states.

Another difference between the quantum and the classical emerges from the observation that for non-abelian subsystem algebras it can happen that, although $\mathfrak{N}_{1}$ and $\mathfrak{N}_{2}$ are mutually commuting (and thus meet our minimal requirement for separability/independence), there may not exist any normal product states on $\mathfrak{N}_{1} \vee \mathfrak{N}_{2}$. An example from the quantization of the Klein-Gordon field is provided by the algebras $\mathfrak{N}\left(\mathcal{W}_{L}\right)$ and $\mathfrak{N}\left(\mathcal{W}_{R}\right)$ associated respectively with left and right Rindler wedge regions $\mathcal{W}_{L}$ and $\mathcal{W}_{R}$ of Minkowski spacetime. ${ }^{9}$ Since $\mathcal{W}_{L}$ and $\mathcal{W}_{R}$ are relatively spacelike and microcausality holds for the Klein-Gordon field, $\left[\mathfrak{N}\left(\mathcal{W}_{L}\right), \mathfrak{N}\left(\mathcal{W}_{R}\right)\right]=0$; and yet because of the cluster property of the vacuum, there are no normal product states on $\mathfrak{N}\left(\mathcal{W}_{L}\right) \vee \mathfrak{N}\left(\mathcal{W}_{R}\right) .{ }^{10}$ (Note that the qualification 'normal' is essential since there are non-normal product states on $\mathfrak{N}\left(\mathcal{W}_{L}\right) \vee \mathfrak{N}\left(\mathcal{W}_{R}\right)$.) On the standard definition of quantum entanglement discussed in Section 4 it follows that every normal state on $\mathfrak{N}\left(\mathcal{W}_{L}\right) \vee \mathfrak{N}\left(\mathcal{W}_{R}\right)$ is quantum entangled over the subsystem algebras.

This last example raises an issue about how to understand entanglement: Assuming that the physically realizable states coincide with the normal states, does the fact a system algebra $\mathfrak{N}_{1} \vee \mathfrak{N}_{2}$ does not admit any normal product states over $\mathfrak{N}_{1}$ and $\mathfrak{N}_{2}$ mean that (a) entanglement over $\mathfrak{N}_{1}$ and $\mathfrak{N}_{2}$ is necessary/essential since all physically realizable states are entangled (indeed, quantum entangled), or does it mean that (b) $\mathfrak{N}_{1}$ and $\mathfrak{N}_{2}$, despite 
their mutual commutativity, are not sufficiently separable/independent for the question of entanglement to be properly raised? Choosing option (b) involves opening the Pandora's box of separability/independence requirements studied in the mathematical physics literature. ${ }^{11} \mathrm{I}$ will only mention here one of the strongest of these separability/independence requirements:

Def. 2 The mutually commuting von Neumann algebras $\mathfrak{N}_{1}$ and $\mathfrak{N}_{2}$ satisfy the split property iff there exist a Type I factor $\mathfrak{F}$ such that $\mathfrak{N}_{1} \subset \mathfrak{F} \subset \mathfrak{N}_{2}^{\prime}$.

The split property implies that $\mathfrak{N}_{1} \vee \mathfrak{N}_{2}$ is $*$-isomorphic to the tensor product algebra $\mathfrak{N}_{1} \bar{\otimes} \mathfrak{N}_{2}$ and, thus, that there are normal product states aplenty. ${ }^{12}$ (It follows that $\mathfrak{N}\left(\mathcal{W}_{L}\right) \vee \mathfrak{N}\left(\mathcal{W}_{R}\right)$ does not have a tensor product structure and that $\mathfrak{N}\left(\mathcal{W}_{L}\right)$ and $\mathfrak{N}\left(\mathcal{W}_{R}\right)$, although mutually commuting, are not split.) For the Klein-Gordon field the split property has been established for some local algebras associated with relatively spacelike regions, in particular, strictly spacelike separated double cone regions. ${ }^{13}$ But the split property can fail for the algebras associated with relatively spacelike but unbounded regions, e.g. Rindler wedge regions. In ordinary QM the split property automatically holds if, as is tacitly assumed, the algebras associated with distinguishable particles are mutually commuting Type I factors; indeed, $\mathfrak{B}\left(\mathcal{H}_{1}\right) \vee \mathfrak{B}\left(\mathcal{H}_{2}\right) \simeq$ $\mathfrak{B}\left(\mathcal{H}_{1}\right) \overline{\bar{B}} \mathfrak{B}\left(\mathcal{H}_{2}\right) \simeq \mathfrak{B}\left(\mathcal{H}_{1}\right) \otimes \mathfrak{B}\left(\mathcal{H}_{2}\right) \simeq \mathfrak{B}\left(\mathcal{H}_{1} \otimes \mathcal{H}_{2}\right)$

The implicit assumption in most of the philosophical literature, and a good deal of the physics literature on quantum computing and quantum information theory, that the composite system algebra has the structure of tensor product of Type I factors is to be deplored because it neglects possibilities that need to be explored. But to simplify the discussion below, I will operate with a tensor product structure for system algebras.

\section{What Is Quantum Entanglement?}

Entanglement (E0) in the sense of Def. 1(i) can obtain even when the subsystem algebras $\mathfrak{N}_{1}$ and $\mathfrak{N}_{2}$ are classical (abelian), although as noted above the entangled states must be mixed. If, however, $\mathfrak{N}_{1}$ and $\mathfrak{N}_{2}$ are quantum (non-abelian) then pure states can be entangled, and no one doubts that these states are examples of quantum entanglement. But for mixed states on non-abelian subsystem algebras it is far from clear that (E0) captures 
genuine quantum entanglement. Consider, for example, the system algebra $\mathfrak{B}\left(\mathcal{H}_{1}\right) \otimes \mathfrak{B}\left(\mathcal{H}_{2}\right)$ and a normal mixed state corresponding to a density operator of the form

$$
\frac{1}{2} E_{\left|\eta_{1}\right\rangle} \otimes E_{\left|\xi_{1}\right\rangle}+\frac{1}{2} E_{\left|\eta_{2}\right\rangle} \otimes E_{\left|\xi_{2}\right\rangle}
$$

where the $E_{\left|\eta_{i}\right\rangle}$ and $E_{\left|\eta_{j}\right\rangle}$ are respectively the projectors onto the rays spanned by the vectors $\left|\eta_{i}\right\rangle \in \mathcal{H}_{1}$ and $\left|\xi_{j}\right\rangle \in \mathcal{H}_{2}$ chosen from an ON basis $\left\{\left|\eta_{i}\right\rangle \otimes\left|\xi_{j}\right\rangle\right\}$ for $\mathcal{H}_{1} \otimes \mathcal{H}_{2}$. This state can be prepared by flipping a fair classical coin and then preparing the system in the vector state corresponding to $\left|\eta_{1}\right\rangle \otimes\left|\xi_{1}\right\rangle$ or (classical or!) to $\left|\eta_{2}\right\rangle \otimes\left|\xi_{2}\right\rangle$ according as the coin lands Heads or lands Tails. On either alternative the state chosen by the coin flip is non-entangled in sense (E0) and, thus, the entanglement of the mixed state is due entirely to ignorance about the outcome of the coin flip.

Of course, one might question why mixed states need to be considered. One answer is that the systems we deal with are rarely isolated from a larger environment. Interaction with the environment results in entanglement (E0), and this entanglement results in subsystem states that are mixed. In fact, it is not hard to establish the following

Proposition: If $\mathfrak{N}_{1}$ and $\mathfrak{N}_{2}$ are mutually commuting and $\omega$ is a pure normal non-product state on $\mathfrak{N}_{1} \vee \mathfrak{N}_{2}$, then the subsystem states obtained by the restrictions $\left.\omega\right|_{\mathfrak{N}_{1}}$ and $\left.\omega\right|_{\mathfrak{N}_{2}}$ of $\omega$ to the subsystem algebras are both mixed.

So even if we deal only with pure states at the composite system level, mixed states will out at the subsystem level. This point is made in spades in relativistic QFT where the issue of quantum entanglement of local algebras arises only for mixed states, at least insofar as only normal states are physically realizable. ${ }^{14}$ Moreover, on the criteria of quantum entanglement considered in this Section and the next, mixed states can induce genuine quantum entanglement, even of the deepest kind.

Returning to the initial example, the state corresponding to the density operator (1) might not have resulted from a classical coin flipping mechanism. For example, it might arise from restricting the vector state $\omega_{|\Xi\rangle}$, with $|\Xi\rangle=\sum_{m=1}^{2} \alpha_{m}\left|\eta_{m}\right\rangle \otimes\left|\xi_{m}\right\rangle \otimes\left|\varsigma_{m}\right\rangle,\left|\alpha_{1}\right|^{2}=\left|\alpha_{2}\right|^{2}=1 / 2$, on the tri-partite 
algebra $\mathfrak{B}\left(\mathcal{H}_{1}\right) \otimes \mathfrak{B}\left(\mathcal{H}_{2}\right) \otimes \mathfrak{B}\left(\mathcal{H}_{3}\right)$ to the bi-partite $\mathfrak{B}\left(\mathcal{H}_{1}\right) \otimes \mathfrak{B}\left(\mathcal{H}_{2}\right)$, where $\left\{\left|\eta_{i}\right\rangle \otimes\left|\xi_{j}\right\rangle \otimes\left|\varsigma_{k}\right\rangle\right\}$ is an $\mathrm{ON}$ basis for $\mathcal{H}_{1} \otimes \mathcal{H}_{2} \otimes \mathcal{H}_{3} \cdot{ }^{15}$ However, most of the extant research on entanglement is focused on finding a formal criterion of quantum entanglement, the implicit presupposition being that, regardless of the actual facts about how the state arises, the mere possibility of the kind of classically instrumented state preparation illustrated in the above coin flipping example disqualifies a state as inducing genuine quantum entanglement. ${ }^{16}$ This attitude suggests the following definition.

Def. 3. (i) Let $\mathfrak{N}_{1}$ and $\mathfrak{N}_{2}$ be mutually commuting von Neumann algebras acting on a common Hilbert space. A normal state $\omega$ on $\mathfrak{N}_{1} \bar{\otimes} \mathfrak{N}_{2}$ is decomposable ${ }^{17}$ with respect to $\mathfrak{N}_{1}$ and $\mathfrak{N}_{2}$ iff it can be approximated by convex linear combinations of normal product states with respect to $\mathfrak{N}_{1}$ and $\mathfrak{N}_{2}$, i.e. $\omega$ lies in the norm closure $^{18}$ of the hull of convex linear combinations of normal product states. $^{19}$

(ii) $\omega$ induces quantum entanglement with respect to $\mathfrak{N}_{1}$ and $\mathfrak{N}_{2}$ in the standard sense (E1) iff it is indecomposable.

I have been unable to determine when this definition was first proposed, but it is certainly around in the late 1980s (see Raggio 1988 and Werner 1989). Dubbing it the standard sense of quantum entanglement seems justified since, despite the fact that it is not often explicitly enunciated, I believe that it makes the best sense of pronouncements on quantum entanglement in the post-Bell physics literature; but establishing this claim would require a literature review that I cannot undertake here. Since different convex linear combinations can produce the same mixed state, it is not easy to determine whether or not a given mixed state is indecomposable. ${ }^{20}$ (The reader may wish to try the following exercise: Look ahead to formula $\left(4^{\prime}\right)$ of Section 6 which presents a one-parameter family of density operators. For which values of the parameter $\alpha$ are the corresponding states decomposable?)

If one of the goals of providing a criterion of quantum entanglement is to find what distinguishes the quantum from the classical, then the claim that (E1) fills the bill is buttressed by a result due to Raggio (1988):

Theorem. Let $\mathfrak{N}_{1}$ and $\mathfrak{N}_{2}$ be mutually commuting von Neumann algebras acting on a common Hilbert space. Then the following two conditions are equivalent: 
(R1) At least one of $\mathfrak{N}_{1}$ and $\mathfrak{N}_{2}$ is abelian

(R2) No normal state on $\mathfrak{N}_{1} \bar{\otimes} \mathfrak{N}_{2}$ is (E1) entangled over $\mathfrak{N}_{1}$ and $\mathfrak{N}_{2}$.

Putting the result in the contrapositive mode, the possibility of (E1) entanglement over the subsystem algebras $\mathfrak{N}_{1}$ and $\mathfrak{N}_{2}$ arises just in case both algebras are non-classical (= nonabelian). ${ }^{21}$ With a bit of charity one could take this as a vindication of Schrödinger's claim quoted in the Introduction; a somewhat less charitable view is taken in Section 9.

Do we now know what quantum entanglement is? We know part of the story. In the next Section we will meet considerations that militate in favor of setting the bar for quantum entanglement higher than (E1).

\section{Stronger Notions of Quantum Entanglement}

Part of the motivation for the definition of (E1) was to rule out cases of states where the entanglement (E0) could be due the ignorance of a classical mechanism that generates mixture weights over non-entangled states. But if this is a valid concern then one should also worry about more subtle ways that the entanglement could be generated by a classical mechanism. Here is one way. A hidden variable (hv) model of a state $\omega$ on $\mathfrak{N}_{1} \bar{\otimes} \mathfrak{N}_{2}$ acting on $\mathcal{H}_{1} \otimes \mathcal{H}_{2}$ contains the following ingredients. First, a probability space $(\Lambda, d \mu)$ where $d \mu$ is a countably additive probability measure on a $\sigma$-algebra of subsets of $\Lambda$, the hidden variable space. Next, for projections $E \in \mathfrak{N}_{1}$ and $F \in \mathfrak{N}_{2}$ there are measurable "response functions" $\mathcal{F}_{E}: \Lambda \rightarrow[0,1]$ and $\mathcal{F}_{F}: \Lambda \rightarrow[0,1]$, interpreted as giving the probability for a Yes outcome of a measurement of $E$ and of $F$ respectively when the hidden state takes value $\lambda \in \Lambda .{ }^{22}$ And, finally, the model must recover the quantum probabilities in that for all projections $E \in \mathfrak{N}_{1}$ and $F \in \mathfrak{N}_{2}$

$$
\omega(E \otimes F)=\int_{\Lambda} \mathcal{F}_{E}(\lambda) \mathcal{F}_{F}(\lambda) d \mu(\lambda) .
$$

If such a model is in operation the entanglement (if any) of $\omega$ is due to ignorance of the value of the hidden state. But whether or not such a model is actually in operation, the fact that it is mathematically possible and could be in operation might, in parallel with the motivation for (E1), be taken to disqualify $\omega$ as engendering genuine quantum entanglement. This sentiment is codified in 
Def. 4 A normal state $\omega$ on $\mathfrak{N}_{1} \bar{\otimes} \mathfrak{N}_{2}$ induces quantum entanglement with respect to $\mathfrak{N}_{1}$ and $\mathfrak{N}_{2}$ in the no hidden variable sense (E2) iff it does not admit a hv model.

As the reader might guess, (E2) is strictly stronger than (E1). That (E1) $\nRightarrow($ E2) is shown by a sub-class of the Werner states (Werner 1989) with the property that they can give quantum entanglement (E1) even though they admit a hv model. These states will be considered in more detail in the following Section. To see that $(\mathrm{E} 2) \Rightarrow(\mathrm{E} 1)$ suppose that $\neg(\mathrm{E} 1)$ and show how to construct a hv model. If the normal state $\omega$ violates (E1) then it can be approximated in the norm by convex linear combinations of normal states corresponding to density operators on $\mathcal{H}_{1} \otimes \mathcal{H}_{2}$, the Hilbert space on which $\mathfrak{N}_{1} \bar{\otimes} \mathfrak{N}_{2}$ acts. This means that there is a measure $d \mu$ on $\Lambda:=\mathcal{D}_{1} \mathrm{X} \mathcal{D}_{2}$, where $\mathcal{D}_{1}$ and $\mathcal{D}_{2}$ are the spaces of density operators on $\mathcal{H}_{1}$ and $\mathcal{H}_{2}$ respectively, such that for all projections $E \in \mathfrak{N}_{1}$ and $F \in \mathfrak{N}_{2}$

$$
\begin{aligned}
\omega(E \otimes F) & =\int_{\Lambda}\left(\varphi_{\rho_{1}} \otimes \varphi_{\rho_{2}}\right)(E \otimes F) d \mu\left(\rho_{1}, \rho_{2}\right) \\
& =\int_{\Lambda} \varphi_{\rho_{1}}(E) \varphi_{\rho_{2}}(F) d \mu\left(\rho_{1}, \rho_{2}\right)
\end{aligned}
$$

where $\varphi_{\rho_{1}}$ and $\varphi_{\rho_{2}}$ are the normal states corresponding to the density operators $\rho_{1}$ and $\rho_{2}$ respectively. This is a hv model with the response functions $\mathcal{F}_{E}\left(\rho_{1}, \rho_{2}\right)=\varphi_{\rho_{1}}(E)$ and $\mathcal{F}_{F}\left(\rho_{1}, \rho_{2}\right)=\varphi_{\rho_{2}}(F)$.

As is well known, the issue of hidden variable models is closely related to the Bell inequalities. Bell operators for $\mathfrak{N}_{1} \bar{\otimes} \mathfrak{N}_{2}$ have the form $B:=$ $\frac{1}{2}\left[X_{1} \otimes\left(Y_{1}+Y_{2}\right)+X_{2} \otimes\left(Y_{1}-Y_{2}\right)\right]$ where the $X_{i} \in \mathfrak{N}_{1}$ and $Y_{j} \in \mathfrak{N}_{2}, i, j=1,2$, are self-adjoint and $-I \leq X_{i}, Y_{j} \leq I$. For a state $\omega$ on $\mathfrak{N}_{1} \bar{\otimes} \mathfrak{N}_{2}$ define $\beta(\omega):=\sup _{B}|\omega(B)|$. A state $\omega$ such that $\beta(\omega)>1$ is said to violate the Bell inequalities, with maximal violation occurring when $\beta(\omega)=\sqrt{2}$.

Now if the Raggio theorem can be used to motivate taking (E1) to be the correct explication of quantum entanglement, then it can equally well be taken to motivate the following alternative criterion of quantum entanglement:

Def. 4 A normal state $\omega$ on $\mathfrak{N}_{1} \bar{\otimes} \mathfrak{N}_{2}$ induces quantum entanglement in the Bell sense (E3) with respect to $\mathfrak{N}_{1}$ and $\mathfrak{N}_{2}$ iff it violates the Bell inequalities. 
For Raggio's theorem not only showed that (R1) is equivalent to (R2) but also that $(\mathrm{R} 1)$ is equivalent to

(R3) No normal state on $\mathfrak{N}_{1} \bar{\otimes} \mathfrak{N}_{2}$ induces (E3) entanglement with respect to $\mathfrak{N}_{1}$ and $\mathfrak{N}_{2}$.

So the possibility of (E3) entanglement over $\mathfrak{N}_{1}$ and $\mathfrak{N}_{2}$ arises just in case both are non-abelian.

The status of (E3) can be further explored by its relationships to (E1) and (E2). In fact, (E3) is strictly stronger than (E2). That (E3) $\Rightarrow(\mathrm{E} 2)$ is one version of the many versions of "Bell's theorem." That (E2) $\nRightarrow$ (E3) was shown by counterexample by Garg and Mermin (1982).

The upshot is that the conditions for entanglement considered so far form a strict hierarchy:

$$
(\mathrm{E} 3) \Rightarrow(\mathrm{E} 2) \Rightarrow(\mathrm{E} 1) \Rightarrow(\mathrm{E} 0)
$$

with none of the arrows being reversible.

\section{Is (E1) Sufficient for Genuine Quantum Entanglement? Is It Necessary?}

Is rising to the level of (E1) in the hierarchy sufficient to ensure genuine quantum entanglement? One way to explore this issue is in terms of Werner states (Werner 1989), which are constructed so that they admit a hv model and, therefore, satisfy the Bell inequalities. For the algebra $\mathfrak{B}\left(\mathbb{C}^{2}\right) \otimes \mathfrak{B}\left(\mathbb{C}^{2}\right)$ acting on $\mathbb{C}^{2} \otimes \mathbb{C}^{2}$ an example of a Werner state is the normal mixed state corresponding to density operator

$$
\frac{1}{2} \frac{I \otimes I}{4}+\frac{1}{2} E_{\left|\Psi_{S}\right\rangle}
$$

where $E_{\left|\Psi_{S}\right\rangle}$ is the projection onto the ray spanned by a "singlet" state vector $\left|\Psi_{S}\right\rangle \in \mathbb{C}^{2} \otimes \mathbb{C}^{2}$. To construct such a vector, choose an orthonormal basis $\left|\eta_{i}\right\rangle$ for $\mathbb{C}^{2}$ and set $\left|\Psi_{S}\right\rangle:=\frac{1}{\sqrt{2}}\left[\left|\eta_{1}\right\rangle \otimes\left|\eta_{2}\right\rangle-\left|\eta_{2}\right\rangle \otimes\left|\eta_{1}\right\rangle\right]$. The vector state on $\mathfrak{B}\left(\mathbb{C}^{2}\right) \otimes \mathfrak{B}\left(\mathbb{C}^{2}\right)$ corresponding to $\left|\Psi_{S}\right\rangle$ gives a maximal violation of the Bell inequalities.

Now the state corresponding to (4) can be produced by flipping a fair classical coin and preparing the state corresponding to $\frac{I \otimes I}{4}$ or (classical 
or!) to $E_{\left|\Psi_{S}\right\rangle}$ according as the coin lands Heads or lands Tails. $50 \%$ of the time the state prepared is not entangled at all and the other $50 \%$ of the time the state prepared induces entanglement that gives a maximal violation of the Bell inequalities. In those cases where we don't know the result of the coin flip, does the entanglement of the resulting mixed state represent genuine quantum entanglement? If you were queasy about seeing genuine quantum entanglement when the entanglement disappears in $100 \%$ of the cases however the classical uncertainty is resolved - as in the case of the state corresponding to (1) when prepared by a classical coin flip - should you likewise be queasy when the entanglement could disappear altogether in $50 \%$ of the cases when the classical uncertainty is resolved? ${ }^{23}$

If you answer Yes then you may want to set the threshold for genuine quantum entanglement higher than (E1), for the state corresponding to (4) is indecomposable. One way to do this is to move up in the hierarchy surveyed in the preceding Section. Moving up to (E2) would do the job here since (4) admits a hv model. Another way would be to step outside the hierarchy, e.g. by strengthening Def. 3 to exclude states that are partially decomposable in that they can be approximated in the norm by convex linear combinations of normal states some but not necessarily all of which are product states. ${ }^{24}$

If you answer No on the grounds that, even though a classical mechanism can set the mixture weights, any mixture involving a non-trivial contribution from a state giving a maximal violation of the Bell inequalities should be counted as quantum entangled, then you are committed to saying that for any value of $0 \leq \alpha<1$ the state corresponding to

$$
\alpha \frac{I \otimes I}{4}+(1-\alpha) \frac{1}{2} E_{\left|\Psi_{S}\right\rangle}
$$

is quantum entangled. Consequently, you must abandon indecomposability as a necessary condition for quantum entanglement since for any value of $\alpha>2 / 3$ the corresponding Werner state is decomposable. But you need not answer No on these grounds. You could maintain that a state corresponding to $\left(4^{\prime}\right)$ gives rise to genuine quantum entanglement on the grounds that this entanglement can be used to perform imperfect but better than classical teleportation for values of $\alpha \leq 2 / 3$, which is precisely the range where indecomposability obtains (Popescu 1994).

Perhaps in the end a pragmatic attitude is best: rather than haggle over which is the correct explication of quantum entanglement, simply recognize that there are a variety of interrelated ways to think about quantum entan- 
glement, and in investigating some aspect of quantum systems seize on the explication of quantum entanglement that promotes the most fruitful results.

\section{Quantum Discord}

Quantum information theorists have noted that some decomposable (and, therefore, non-(E1)) states can display non-classical features, a phenomenon they dub "quantum discord." This discord might be thought to signal a form of quantum entanglement that lies between simple entanglement (E0) and the standard sense of quantum entanglement (E1).

The first step to understanding quantum discord is to distinguish a subset of decomposable states dubbed "classical," the basic idea being that "classical" states allow acquisition of information via measurements on the component systems without disturbing the composite state whereas "quantum" states do not make such an allowance (see Ollivier and Zurek 2002, Luo 2008, and Modi et al. 2010). To explain this idea it is helpful to realize that most of the quantum information literature assumes that the observable algebra of composite system has a tensor product structure with the component system algebras being Type I factors - for a bipartite system, the composite system algebra is $\mathfrak{B}\left(\mathcal{H}_{1} \otimes \mathcal{H}_{2}\right)=\mathfrak{B}\left(\mathcal{H}_{1}\right) \otimes \mathfrak{B}\left(\mathcal{H}_{2}\right)$ acting on $\mathcal{H}_{1} \otimes \mathcal{H}_{2}$. In addition, only normal states are ever considered. A resolution of the identity operator $I_{\mathcal{H}_{1}}$ on $\mathcal{H}_{1}$ (respectively, $I_{\mathcal{H}_{2}}$ on $\mathcal{H}_{2}$ ) consists of a set of mutually orthogonal projectors $\left\{E_{i}^{1}\right\} \in \mathcal{H}_{1}$ (respectively, $\left\{E_{j}^{2}\right\} \in \mathcal{H}_{2}$ ) such that $\sum_{i} E_{i}^{1}=I_{\mathcal{H}_{1}}$ (respectively, $\sum_{i} E_{j}^{1}=I_{\mathcal{H}_{2}}$ ). (When no confusion results, the subscripts in the identity operators will be omitted.) Such a resolution is minimal iff all the projectors in the resolution are minimal, i.e. they are one-dimensional. A normal state $\omega$ on $\mathfrak{B}\left(\mathcal{H}_{1}\right) \otimes \mathfrak{B}\left(\mathcal{H}_{2}\right)$ is said to be classical just in case there are minimal resolutions of identities $\left\{E_{i}^{1}\right\}$ and $\left\{E_{j}^{2}\right\}$ such that the corresponding complete projective measurement leaves the density operator $\varrho_{\omega}$ corresponding to $\omega$ invariant in the sense that $\varrho_{\omega}=\sum_{i, j}\left(E_{i}^{1} \otimes E_{j}^{2}\right) \varrho_{\omega}\left(E_{i}^{1} \otimes E_{j}^{2}\right)$ (Luo 2008). ${ }^{25}$ As Ollivier and Zurek (2002) put it, when the state is classical one "can interrogate just one part of a composite system and discover its state while leaving the overall density matrix (as perceived by observers who do not have access to the measurement outcome) unaltered" (p. 3). Non-classical states are deemed "truly quantum" (Luo 2008, p. 2). Note that the set of 
classical states so defined is not closed under convex linear combinations so that classical mixing of classical states can produce non-classical states, a somewhat counterintuitive property.

It was shown by Luo (2008) that a normal state $\omega$ on $\mathfrak{B}\left(\mathcal{H}_{1}\right) \otimes \mathfrak{B}\left(\mathcal{H}_{2}\right)$ is classical just in case $\varrho_{\omega}=\sum_{i, j} p_{i j} E_{i}^{1} \otimes E_{j}^{2}$ for some minimal resolutions $\left\{E_{i}^{1}\right\}$ and $\left\{E_{j}^{2}\right\}$ respectively of the identities $I_{\mathcal{H}_{1}}$ and $I_{\mathcal{H}_{2}}$ and some bipartite probability distribution $\left\{p_{i j}\right\}$. Obviously then, a classical state is decomposable. But but the converse is not true, for in the density matrix representation of a general decomposable state the projection operators in the decomposition do not have to be orthogonal. Concrete examples can be constructed from the class of Werner states on $\mathfrak{B}\left(\mathbb{C}^{2}\right) \otimes \mathfrak{B}\left(\mathbb{C}^{2}\right)$ corresponding to the density operators in $\left(4^{\prime}\right)$. For any value of $\alpha<1$ the resulting state is non-classical, but for $\alpha>2 / 3$ the resulting state is decomposable.

A further clarification of the relation between decomposable and classical states was given by Li and Luo (2008) who showed that decomposable states are obtained as reductions of classical states on higher dimensional Hilbert spaces. More precisely, a normal state $\omega$ on $\mathfrak{B}\left(\mathcal{H}_{1}\right) \otimes \mathfrak{B}\left(\mathcal{H}_{2}\right)$ is decomposable iff there is a classical normal state $\sigma$ on $\mathfrak{B}\left(\mathcal{K}_{1} \otimes \mathcal{H}_{1}\right) \otimes \mathfrak{B}\left(\mathcal{H}_{2} \otimes \mathcal{K}_{2}\right)$, where $\mathcal{K}_{1}$ and $\mathcal{K}_{2}$ are auxiliary Hilbert spaces, such that $\rho_{\omega}=\operatorname{Tr}_{\mathcal{K}_{1} \otimes \mathcal{K}_{2}} \rho_{\sigma}$, where $\operatorname{Tr}_{\mathcal{K}_{1} \otimes \mathcal{K}_{2}}$ denotes the partial trace over $\mathcal{K}_{1} \otimes \mathcal{K}_{2}$.

A non-entangled (i.e. product) normal state $\omega$ on $\mathfrak{B}\left(\mathcal{H}_{1}\right) \otimes \mathfrak{B}\left(\mathcal{H}_{2}\right)$ is classical and, thus by contraposition, a non-classical (NC) state is entangled (E0) ${ }^{26}$ And since a classical state is necessarily decomposable, we have

$$
(\mathrm{E} 1) \Rightarrow(\mathrm{NC}) \Rightarrow(\mathrm{E} 0)
$$

where the arrows are non-reversible. ${ }^{27}$ So formally at least quantum discord can be used to extend the entanglement hierarchy mapped out in the preceding Section. It remains to ask, however, whether (NC) deserves a place in the entanglement hierarchy. Granted that (NC) captures an interesting feature of quantum entanglement, if entanglement there be. But does a non(E1) but (NC) state deserve to be called quantum entangled? Evidence for a positive answer would have to come from a demonstration that non-(E1) but $(\mathrm{NC})$ states can exhibit features associated with quantum entanglement, e.g. teleportation. As far as I am aware the evidence is not forthcoming. 
It is also worth noting that the formal definitions and results on quantum discord reported above lose their motivation for more general types of von Neumann algebras. Non-Type I algebras lack minimal projectors, and Type III algebras do not contain any finite dimensional projectors. Thus, a complete projective measurement in the above defined sense may not correspond to a measurement of genuine observables (self-adjoint elements of the composite system algebra). Nevertheless, the basic idea behind to the idea that a defining feature of "classical reality" is that it "allows acquisition of information without perturbation of the underlying state" (Ollivier and Zurek 2002, p. 3) can, when suitably construed, be applied to general types of von Neumann algebras. And it can be linked to the other two features of "classical reality," as shown by the following result.

Consider a von Neumann algebra $\mathfrak{N}$ acting on a separable $\mathcal{H}$. The following two conditions of classicality are equivalent:

(Class1) $\mathfrak{N}$ is abelian

(Class2) $\mathfrak{N}$ freely allows interrogation without disturbance in that for any normal state $\omega$ on $\mathfrak{N}$ and any projection $E \in \mathfrak{N}, \omega$ is invariant under a non-selective measurement of $E$, i.e. $\omega=\widetilde{\omega}$ where $\widetilde{\omega}(\bullet)=\omega(E \bullet E)+\omega((I-E) \bullet(I-E))$ is the new state induced by the non-selective measurement of $E$ (Lüders rule). ${ }^{28}$

Proof that (Class1) $\Rightarrow$ (Class2): If $\mathfrak{N}$ is abelian it follows that for any $A \in$ $\mathfrak{N}, \widetilde{\omega}(A)=\omega(E A E)+\omega((I-E) A(I-E))=\omega\left(A E^{2}\right)+\omega\left(A(I-E)^{2}\right)=$ $\omega(A E)+\omega(A(I-E))=\omega(A E+A(I-E))=\omega(A(E+I-E))=\omega(A)$.

Proof that (Class2) $\Rightarrow$ (Class1): If $\mathfrak{N}$ freely allows interrogation without disturbance then for any normal state $\omega$, any projection $E \in \mathfrak{N}$ and any $A \in \mathfrak{N}, \omega(A-(E A E+(I-E) A(I-E)))=0$. Choosing $\omega$ to be a faithful state, it follows that $A=E A E+(I-E) A(I-E) .{ }^{29}$ Collecting terms gives $2 E A E=A E+E A$. Multiplying on the right by $E$ results in $E A E=A E$, while multiplying on the left by $E$ results in $E A E=E A$. Together these imply that $A E=E A$, i.e. every element of $\mathfrak{N}$ commutes with every projector of $\mathfrak{N}$. Since a von Neumann algebra is generated by its projectors, this implies that $\mathfrak{N}$ is abelian.

Combining this elementary result with Raggio's deep theorem, the circle of the three ways to make the classical/quantum cut can be closed: 
For $\mathfrak{N}=\mathfrak{N}_{1} \bar{\otimes} \mathfrak{N}_{2}$, both subsystem algebras $\mathfrak{N}_{1}$ and $\mathfrak{N}_{2}$ are nonabelian iff neither subsystem freely admits interrogation without disturbance iff $\mathfrak{N}$ admits normal states that are quantum entangled in the sense of (E1) over $\mathfrak{N}_{1}$ and $\mathfrak{N}_{2}$.

And, again by Raggio's theorem, (E3) can be substituted for (E1).

\section{How Ubiquitous Is Quantum Entanglement?}

The answer to the question that forms the title of this subsection varies depending upon whether the setting is ordinary QM or relativistic QFT.

It is known that for the simple bi-partite qubit algebra $\mathfrak{B}\left(\mathbb{C}^{2}\right) \otimes \mathfrak{B}\left(\mathbb{C}^{2}\right)$ every pure non-product normal state violates the Bell inequalities (Gisin 1991) and, thus, every pure non-product normal state induces quantum entanglement in all three senses (E1)-(E3) over these tensor product algebras. ${ }^{30}$ This result generalizes to higher dimensional Hilbert spaces (Gisin and Peres 1992). Thus, for the types of bi-partite algebras encountered in ordinary QM the only normal entangled states (E0) to escape quantum entanglement are mixed states. But mixed states on these algebras can also produce quantum entanglement; indeed, they can violate the Bell inequalities and even give maximal violations (Braunstein et al. 1992). ${ }^{31}$

However, within the setting of ordinary QM there is reason to wonder whether the subsystems that we - qua limited, finite observers - encounter will exhibit quantum entanglement, either in the standard sense (E1) or one of the stronger varieties. To make this more precise, suppose that the algebra of a typical bi-partite system we encounter has a tensor product structure $\mathfrak{B}\left(\mathcal{H}_{1}\right) \otimes \mathfrak{B}\left(\mathcal{H}_{2}\right)$ and is a subsystem of a larger multi-partite system whose algebra also has the structure $\mathfrak{B}\left(\mathcal{H}_{1}\right) \otimes \mathfrak{B}\left(\mathcal{H}_{2}\right) \otimes \ldots \otimes \mathfrak{B}\left(\mathcal{H}_{N}\right)$, $N \geq 3$, of a tensor product of Type I factors. How effectively does quantum entanglement at the multi-partite level "trickle down" to the bi-partite level? More precisely, suppose that the state of the multi-partite system algebra is a pure (equivalently, vector) state with a generating vector $|\Psi\rangle$ that is not a product state over any pair of its subsystem algebras. Then the reduced state of any bi-partite subsystem will be a mixed entangled (E0) state. But will the reduced state typically be quantum entangled? If the multipartite system state vector can be written in a generalized Schmidt form, i.e.

$|\Psi\rangle=\sum_{j=1} \alpha_{j}\left|x_{j}\right\rangle_{1} \otimes\left|x_{j}\right\rangle_{2} \otimes \ldots \otimes\left|x_{j}\right\rangle_{N}$, where the $\left|x_{j}\right\rangle_{1} \otimes\left|x_{j}\right\rangle_{2} \otimes \ldots \otimes\left|x_{j}\right\rangle_{N}$ come 
from an $\mathrm{ON}$ basis for $\mathcal{H}_{1} \otimes \mathcal{H}_{2} \otimes \ldots \otimes \mathcal{H}_{N}$, then the reduced bi-partite state will be decomposable and, indeed, classical in the sense of Section $7 .{ }^{32}$ However, not every multi-partite state vector can be written in generalized Schmidt form. In the bi-partite case $\mathfrak{B}\left(\mathcal{H}_{1}\right) \otimes \mathfrak{B}\left(\mathcal{H}_{2}\right)$ the Schmidt bi-orthogonal decomposition theorem shows that for any $|\Psi\rangle \in \mathcal{H}_{1} \otimes \mathcal{H}_{2}$ there are ON bases $\left|x_{i}\right\rangle_{1}$ and $\left|y_{j}\right\rangle_{2}$ for $\mathcal{H}_{1}$ and $\mathcal{H}_{2}$ respectively such that $|\Psi\rangle=\sum_{i=1}^{r} \lambda_{i}\left|x_{i}\right\rangle_{1} \otimes\left|y_{i}\right\rangle_{2}$, where the $\lambda_{i}>0$ are real numbers such that $\sum_{i=1}^{r} \lambda_{i}^{2}=1$ (Schmidt 1906). But in the simplest tri-partite case $\mathfrak{B}\left(\mathbb{C}^{2}\right) \otimes \mathfrak{B}\left(\mathbb{C}^{2}\right) \otimes \mathfrak{B}\left(\mathbb{C}^{2}\right)$ there are vectors in the qubit space $\mathbb{C}^{2} \otimes \mathbb{C}^{2} \otimes \mathbb{C}^{2}$ that do not admit a generalized Schmidt decomposition and, moreover, those that do admit such a decomposition are highly non-generic (see Peres 1995).

This is not the end of the story, however; for having a generalized Schmidt decomposition is not necessary for the reduced state of a bi-partite subsystem to be decomposable. For example, Acin et al. (2000) show that for the tripartite qubit system $\mathfrak{B}\left(\mathbb{C}^{2}\right) \otimes \mathfrak{B}\left(\mathbb{C}^{2}\right) \otimes \mathfrak{B}\left(\mathbb{C}^{2}\right)$ every state vector can be written in the form $\lambda_{1}|0\rangle \otimes|0\rangle \otimes|0\rangle+\lambda_{2} e^{i \phi}|1\rangle \otimes|0\rangle \otimes|0\rangle+\lambda_{3}|1\rangle \otimes|0\rangle \otimes|1\rangle+\lambda_{4}|1\rangle \otimes$ $|1\rangle \otimes|0\rangle+\lambda_{5}|1\rangle \otimes|1\rangle \otimes|1\rangle$ where $0 \leq \phi \leq \pi$, "0s" and "1s" have been used to label the qubit states, and again the $\lambda$ 's are real numbers whose squares sum to unity. The reduced density operator for the left-most bi-partite subsystem has the form $\lambda_{1}^{2} E_{|0\rangle} \otimes E_{|0\rangle}+\left(\lambda_{2}^{2}+\lambda_{3}^{2}\right) E_{|1\rangle} \otimes E_{|0\rangle}+\left(\lambda_{4}^{2}+\lambda_{5}^{2}\right) E_{|1\rangle} \otimes$ $E_{|1\rangle}$, which is decomposable and classical. In sum, quantum entanglement does not trickle down in this case. If this no-trickle-down result extends to higher dimensional Hilbert spaces in the sense that a generic subset of the system state vectors has the property that the resultant reduced bi-partite subsystem states are decomposable then genuine quantum entanglement will be non-generic in the systems we encounter. ${ }^{33}$ Or at least this is so if the bipartite states we encounter arise by restricting the state on a larger system to the bi-partite subsystem we encounter. In Section 7 the decision was made to repress issues about how a state actually arises in forging a criterion of quantum entanglement; but these issues cannot be repressed when judging how ubiquitous quantum entanglement is. As far as I am aware the trickle down question remains open for ordinary QM.

The situation is somewhat different in QFT. As mentioned above, in the algebraic formulation of relativistic QFT the algebras associated with open regions of Minkowski spacetime are Type III. The only normal states on 
these algebras are mixed, and typically these mixed states are indecomposable. But the latter fact is not happily described as a trickle down effect in the above sense since the global algebra $\mathfrak{N}(\mathcal{M})$ associated with all of Minkowski spacetime $\mathcal{M}$ is not a tensor product of the the algebras $\mathfrak{N}(\mathcal{O})$ of local regions $\mathcal{O} \subset \mathcal{M}$. Nevertheless, we can inquire whether or not the restriction of some state on $\mathfrak{N}(\mathcal{M})$ - say, the vacuum state $\omega_{\text {vac }}$ - to the subalgebra $\mathfrak{N}\left(\mathcal{O}_{1}\right) \vee \mathfrak{N}\left(\mathcal{O}_{2}\right)$ generated by the local algebras $\mathfrak{N}\left(\mathcal{O}_{1}\right)$ and $\mathfrak{N}\left(\mathcal{O}_{2}\right)$, for relatively spacelike $\mathcal{O}_{1}$ and $\mathcal{O}_{2}$, is quantum entangled over the subalgebras. The answer is affirmative in the strongest sense since the Bell inequalities are violated, sometimes maximally. And what is true of the vacuum state is true of an open dense set of vector states (see, for example, Halvorson and Clifton 2000). Quantum entanglement in this setting is truly ubiquitous. ${ }^{34}$

\section{Schrödinger Revisited}

The distinctions drawn above prompt several questions and comments about the characterization of entanglement given in the Schrödinger quotation cited in the Introduction. In the first place, what exactly does Schrödinger mean when he says that after interaction between two subsystems each may lack a "representative of its own"? If "representative" means state in the algebraic sense then Schrödinger was operating with a false presupposition; for if $\omega$ is any state on the system algebra $\mathfrak{N}$ and if $\mathfrak{N}_{1}, \mathfrak{N}_{2} \subset \mathfrak{N}$ are subsystem algebras, then each of the subsystems has its own state, namely the reduced state $\left.\omega\right|_{\mathfrak{N}_{i}}, i=1,2$, obtained by restricting $\omega$ to the subalgebra $\mathfrak{N}_{i}$, and this is so regardless of whether or not $\omega$ entangles $\mathfrak{N}_{1}$ and $\mathfrak{N}_{2}$ and whether or not the entanglement is genuinely quantum. To be fair to Schrödinger, however, it is evident from the quoted passage that he took "representative" to mean a vector state (the phrase he uses is " $\psi$-function") or a pure state, the two sets of states being the same for the Type I factor algebras used in ordinary QM. And his point was that whereas initially the reduced subsystem states may be pure/vector states, it can happen that after interaction neither subsystem state is a pure/vector state.

Although in the quoted passage Schrödinger takes lack of "representatives" for the subsystems as his criterion of entanglement, on the very next page (1935, p. 558) of the article he says that what "constitutes the entanglement" is that the representative of the composite system is not a product state $^{35}$; and he evidently took this second criterion to be equivalent to his 
original criterion. If to the assumption that the composite system algebra is a tensor product of Type I factors is added the further charitable assumption that the state of the composite system is a vector/pure state, then all is well for Schrödinger's characterizations of entanglement; for the lack of subsystem "representatives" (= subsystem states are not vector/pure states) and simple entanglement (= composite system state is not a product state) coincide with each other and with and quantum entanglement (= composite system state is indecomposable).

However, these happy coincidences fall apart when the composite system state is not pure. And Schrödinger's initial characterization of entanglement goes seriously awry when the subsystem algebras are not Type I factors and, consequently, the coincidence of vector states with pure states does not hold. For example, the subsystem algebras are typically Type III in relativistic QFT, and for these algebras all normal states are vector states, whereas no normal state is pure. Thus, if Schrödinger's bellyache about entanglement derives from the non-vector character of the subsystem states, then QFT should cure it since each subsystem does have its own "representative" (vector state). If, however, the bellyache derives from the mixed character of these states, then QFT should make the bellyache worse since the subsystems cannot possess normal pure states. And in either case Schrödinger's no-representatives-of-their own characterization of entanglement yields incorrect results for Type III subsystem algebras; namely, either they are never entangled (since they always have representatives of their own) or they are always entangled (since they never have representatives of their own).

The aim here is not to unfairly criticize Schrödinger for failure to anticipate cases that were well over the horizon when he was writing in 1935 but rather to note that his initial characterization of entanglement has proven to be unfruitful. More fruitful is the characterization of entanglement in terms of indecomposability. In the spirit of charity one could read Schrödinger as groping towards the latter characterization, but one would be hard pressed to find textual evidence to support such a reading.

\section{The Relativity and Ambiguity of Entanglement}

To restate what is obvious from the algebraic approach, talk of entanglement has to be relativized to a specification of subsystems and their associated subalgebras of observables. In the preceding Sections the obvious did 
not need to be stated since it was simply taken for granted that the "correct" specification was provided by some higher authority or else that there is a natural procedure to generate a unique specification. If we do not want to rely on higher authority (who? what?), we need to get a handle on how the system algebra and the subsystem algebras are generated. To keep the discussion as simple as possible I will ignore the complexities about entanglement uncovered in the preceding Sections and will assume that indecomposability (Def. 3) is both necessary and sufficient for quantum entanglement.

\subsection{Bottom-Up and Top-Down}

As the label indicates, the bottom-up approach starts from a delineation of the von Neumann algebras corresponding to subsystems, and the system algebra is identified with the von Neumann algebra generated by the subsystem algebras. This was the approach that was mainly used in preceding Sections. Again as the label indicates, the top-down approach starts by defining the system algebra and then decomposes this algebra into subalgebras corresponding to subsystems. A concrete illustration follows.

Ex. 1. Two spinless particles moving in d-dim Euclidean space $\mathbb{R}^{d}$. Working this example in the top-down mode, the configuration space for the twoparticle system is $\mathbb{R}^{2 d}$. Quantum lore tells us that the appropriate Hilbert space to describe the composite system is $\mathcal{H}=L_{\mathbb{C}}^{2}\left(\mathbb{R}^{2 d}\right)$, the space of square integrable complex-valued functions on the configuration space. If each of the elements of the set of $\Theta$ of all self-adjoint operators (not necessarily bounded) acting on $L_{\mathbb{C}}^{2}\left(\mathbb{R}^{2 d}\right)$ represents a genuine two-particle observable, then the associated von Neumann composite system algebra is $\mathfrak{N}:=\Theta^{\prime \prime}=\mathfrak{B}\left(L_{\mathbb{C}}^{2}\left(\mathbb{R}^{2 d}\right)\right)$. Now notice that $L_{\mathbb{C}}^{2}\left(\mathbb{R}^{2 d}\right)$ is isomorphic to $L_{\mathbb{C}}^{2}\left(\mathbb{R}^{d}\right) \otimes L_{\mathbb{C}}^{2}\left(\mathbb{R}^{d}\right)$ and, hence, the system algebra $\mathfrak{N}$ is isomorphic to $\mathfrak{B}\left(L_{\mathbb{C}}^{2}\left(\mathbb{R}^{d}\right)\right) \otimes \mathfrak{B}\left(L_{\mathbb{C}}^{2}\left(\mathbb{R}^{d}\right)\right) .{ }^{36}$ Thus, there is a natural decomposition of the composite system algebra into the one-particle subsystem algebras - in this case the decomposition takes a tensor product form. The example can be reworked in the bottom-up mode, starting from the one-particle descriptions, $\mathcal{H}_{1}=L_{\mathbb{C}}^{2}\left(\mathbb{R}^{d}\right), \mathfrak{N}_{1}:=\Theta_{1}^{\prime \prime}=\mathfrak{B}\left(\mathcal{H}_{1}\right)$ for particle $\# 1$ and $\mathcal{H}_{2}=L_{\mathbb{C}}^{2}\left(\mathbb{R}^{d}\right), \mathfrak{N}_{2}:=\Theta_{2}^{\prime \prime}=\mathfrak{B}\left(\mathcal{H}_{2}\right)$ for particle $\# 2$, where $\Theta_{i}$ is the set of all self-adjoint operators on $\mathcal{H}_{i}, i=1,2$. If it is demanded that $\mathfrak{N}_{1}$ and $\mathfrak{N}_{2}$ are mutually commuting then the split property is satisfied and, consequently, the composite system algebra $\mathfrak{N}:=\mathfrak{N}_{1} \vee \mathfrak{N}_{2}=\mathfrak{B}\left(\mathcal{H}_{1}\right) \vee \mathfrak{B}\left(\mathcal{H}_{2}\right)$ is *-isomorphic to $\mathfrak{B}\left(L_{\mathbb{C}}^{2}\left(\mathbb{R}^{d}\right)\right) \otimes \mathfrak{B}\left(L_{\mathbb{C}}^{2}\left(\mathbb{R}^{d}\right)\right)$, in agreement with the top-down approach. 
This example - the very one Schrödinger had in mind - is misleading in suggesting that there will always be a unique "natural" or "proper" decomposition of the system algebra. Of course, everything rides on what counts as a "natural" or "proper" decomposition, but before confronting this issue it will be helpful to have another example to give a sense of how the non-uniqueness of decompositions can arise.

Ex. 2: Two-spins system (Zanardi et al. 2004, Harshman and Wichramasekara 2007, and de la Torre et al. 2010). Again starting in the top-down mode, the system algebra $\mathfrak{N}$ acting on $\mathcal{H}=\mathbb{C}^{4}$ is generated by the Pauli spin operators $\Theta=\left\{\sigma_{j}^{x}, \sigma_{j}^{y}, \sigma_{j}^{z}, j=1,2\right\}$, resulting in $\mathfrak{N}:=\Theta^{\prime \prime}=\mathfrak{B}\left(\mathbb{C}^{4}\right) .{ }^{37}$ The most obvious decomposition of $\mathfrak{N}$ into subsystem algebras is $\mathfrak{N}_{1}\left(\mathbb{C}^{2}\right) \otimes \mathfrak{N}_{2}\left(\mathbb{C}^{2}\right)$ where $\mathfrak{N}_{k}=\Theta_{k}^{\prime \prime}$ with $\Theta_{k}:=\left\{\sigma_{k}^{x}, \sigma_{k}^{y}, \sigma_{k}^{z}\right\}, k=1,2$. But other decompositions are possible, e.g. $\widehat{\mathfrak{N}}_{1}\left(\mathbb{C}^{2}\right) \otimes \widehat{\mathfrak{N}}_{2}\left(\mathbb{C}^{2}\right)$ where $\widehat{\mathfrak{N}}_{1}$ and $\widehat{\mathfrak{N}}_{2}$ are the von Neumann algebras generated respectively by $\widehat{\Theta}_{1}=\left\{\sigma_{1}^{x}, \sigma_{1}^{y} \sigma_{2}^{z}, \sigma_{1}^{z} \sigma_{2}^{z}\right\}$ and $\widehat{\Theta}_{2}=\left\{\sigma_{2}^{z}, \sigma_{1}^{x} \sigma_{2}^{y}, \sigma_{1}^{x} \sigma_{2}^{x}\right\}$. It is left to the reader to rework the example in the bottom-up mode.

It should be evident that the very same state on the system algebra of Ex. 2 that is entangled with respect to one of the given decompositions may not be entangled with respect to the other. The moral is that unless constraints are placed on what counts as a subsystem algebra, the notion of entanglement ambiguous. Just how radical the threatened ambiguity is can be illustrated by reflecting on how the bottom-up approach can be mathematically generalized. Start with some Hilbert space $\mathcal{H}$, and choose sets $\Theta_{1}$ and $\Theta_{2}$ of mutually commuting self-adjoint operators acting on $\mathcal{H}$ such that the von Neumann algebras they generate, viz. $\mathfrak{N}_{1}:=\Theta_{1}^{\prime \prime}$ and $\mathfrak{N}_{2}:=\Theta_{2}^{\prime \prime}$, are non-abelian as well as being mutually commuting. Then declare that $\mathfrak{N}_{1}$ and $\mathfrak{N}_{2}$ are subsystem algebras, generating the composite system algebra $\mathfrak{N}:=\mathfrak{N}_{1} \vee \mathfrak{N}_{2}$. (One might want to require that, in addition to mutual commutativity, $\mathfrak{N}_{1}$ and $\mathfrak{N}_{2}$ satisfy some stronger of the other separability/independence requirements, e.g. that $\mathfrak{N}_{1} \vee \mathfrak{N}_{2}$ has a tensor product structure. But decisions on this matter will not affect the main points that follow.) There will always be alternative choices of observables $\widehat{\Theta}_{1}$ and $\widehat{\Theta}_{2}$ generating non-abelian but mutually commuting $\widehat{\mathfrak{N}}_{1}:=\left(\widehat{\Theta}_{1}\right)^{\prime \prime}$ and $\widehat{\mathfrak{N}}_{2}:=\left(\widehat{\Theta}_{2}\right)^{\prime \prime}$, which together generate the same system algebra, i.e. $\widehat{\mathfrak{N}}_{1} \vee \widehat{\mathfrak{N}}_{2} \simeq \mathfrak{N}_{1} \vee \mathfrak{N}_{2}$. If such constructions are unconstrained then entanglement becomes ambiguous since different choices for subalgebras can make entanglement appear or disappear. For if each of the hatted subalge- 
bras "mixes" elements from both of the unhatted subalgebras then a state on the composite system algebra that is unentangled over $\mathfrak{N}_{1}$ and $\mathfrak{N}_{2}$ may be entangled over $\widehat{\mathfrak{N}}_{1}$ and $\widehat{\mathfrak{N}}_{2}$ or vice versa.

The scope of the threat of the ambiguity of entanglement as it arises in the top-down mode is also dire. Call a von Neumann algebra $\mathfrak{N}$ quantum entanglement ready just in case it admits a quantum entanglement ready decomposition in the form of a pair of non-abelian but mutually commuting subalgebras $\mathfrak{N}_{1}$ and $\mathfrak{N}_{2}$ such that $\mathfrak{N}=\mathfrak{N}_{1} \vee \mathfrak{N}_{2}$. If it is required in addition that the decomposition take the form of tensor product then some algebras are not entanglement ready. Suppose that $\mathfrak{N} \simeq \mathfrak{N}_{1} \bar{\otimes} \mathfrak{N}_{2}$ acting on $\mathcal{H} \simeq \mathcal{H}_{1} \otimes$ $\mathcal{H}_{2}$. If $\operatorname{dim}(\mathcal{H})$ is a prime number then either $\operatorname{dim}\left(\mathcal{H}_{1}\right)=1$ or $\operatorname{dim}\left(\mathcal{H}_{2}\right)=1$ and, thus, either $\mathfrak{N}_{1}$ or $\mathfrak{N}_{2}$ is abelian, making quantum entanglement over $\mathfrak{N}_{1}$ or $\mathfrak{N}_{2}$ impossible. Setting aside such cases, I conjecture the following:

(C) Let $\mathfrak{N}$ be any entanglement ready von Neumann algebra and let $\omega$ be any normal state on $\mathfrak{N}$; if there is an entanglement ready decomposition of $\mathfrak{N}$ such that $\omega$ is not quantum entangled for that decomposition then there is another entanglement ready decomposition for which $\omega$ is quantum entangled.

If $(\mathrm{C})$ is true it follows that whenever quantum entanglement is possible, it is possible to consider any normal state to be quantum entangled, i.e. for any entanglement ready $\mathfrak{N}$ and any normal $\omega$ state on $\mathfrak{N}$ there is an entanglement ready decomposition for which $\omega$ is quantum entangled. Even if (C) fails, it should be evident from the examples above and those that follow at the mathematical level there is ambiguity aplenty for entanglement. The physical significance of this mathematical ambiguity is far from evident.

\subsection{Realism vs. Pragmatism}

Many different reactions to the threatened rampant ambiguity of entanglement are possible, but to keep the discussion simple I will focus on two broad categories.

Realism. The Realist claims that the kind of entanglement we should be concerned with is entanglement over subalgebras that correspond to real as opposed to virtual or fictitious subsystems. Obviously, this distinction cannot be drawn in terms of purely formal conditions on the algebras but must come from background physics. The background information might, 
for example, provide grounds for thinking that the system at issue should satisfy a fundamental symmetry, and this in turn would preference decompositions of the system algebra into subalgebras that are invariant under said symmetry (see Harshman and Wickramasekara 2007). The background information may not be sufficient to draw a bright red line between real vs. virtual or fictitious subsystems, but the Realist hopes it is serves to rein in the threatened rampant ambiguity of entanglement. But where the hope is utterly dashed and there is no principled way to draw the distinction, the Realist holds that entanglement ought to be regarded as a moot topic.

Pragmatism. The Pragmatist asserts that there is no metaphysically valid way to draw a line between subsystems that are "real" and those that are "fictitious" or "virtual." Any decomposition of the system algebra into subalgebras meeting appropriate formal criteria is as good a way as any other for defining subsystems. Thus, contrary to Realism, there is no metaphysically valid distinction to be drawn between "real" entanglement (i.e. entanglement over "real" subsystems) and "faux" entanglement (i.e. entanglement over "fictitious" or "virtual" subsystems). For the Pragmatist the only valid distinctions are the ones that can be drawn in terms of considerations of utility. For example, the distinction between decompositions where the observables of the subalgebras are experimentally accessible and those where they are not, a matter that obviously varies with the experimental context. And one might want to further subdivide the experimentally accessible entanglements into those that are useful for, say, performing quantum computations and those that are not, again a distinction that depends on the context.

The way the debate between Realism and Pragmatism plays out can be illustrated in terms of various examples. Revisiting the two-spins example (Ex. 2), the Pragmatist would say both decompositions of the system algebra are equally valid, but that the first mentioned decomposition is favored in contexts where the individual spins are experimentally accessible while the second is favored where spin-spin interactions are accessible. ${ }^{38}$ The Realist would respond to the two-spins example by claiming that we know from a wider context that spin is not a self-subsistent property but a property of particles - the Pauli spin operators $\sigma_{1}^{x}, \sigma_{1}^{y}, \sigma_{1}^{z}$ go together because they are attached to particle $\# 1$ and the operators $\sigma_{2}^{x}, \sigma_{2}^{y}, \sigma_{2}^{z}$ go together because they are attached to particle \#2. From this wider perspective the sets of observables $\left\{\sigma_{1}^{x}, \sigma_{1}^{y} \sigma_{2}^{z}, \sigma_{1}^{z} \sigma_{2}^{z}\right\}$ and $\left\{\sigma_{2}^{z}, \sigma_{1}^{x} \sigma_{2}^{y}, \sigma_{1}^{x} \sigma_{2}^{x}\right\}$ used to generate the nonstandard subalgebras are seen to be gerrymandered and do not correspond 
to genuine subsystems identified by independent means.

Ex. 3: The hydrogen atom. In addition to the obvious way of construing the atom as composed of an electron and a proton, one can also think of it as composed of a particle of mass $M=m_{p}+m_{e}$ moving freely in the center of mass coordinates and another particle of reduced mass $m_{r}^{-1}=m_{p}^{-1}+m_{e}^{-1}$ moving in a fixed Coulomb field. The ground state of the hydrogen atom is entangled with respect to the proton's position and electron's position but is unentangled with respect to the to the center of mass coordinates and the relative proton-electron coordinates (see Tommasini and Timmermans 1998).

The Pragmatist will opine that whether or not to see entanglement in the ground state of the hydrogen atom depends on which position observables are experimentally accessible - the proton and electron positions or the center of mass and relative particle positions. The Realist will respond in much the same fashion as in the previous case; viz. knowledge gained from a wider context informs us that electrons and protons are real particles while center of mass and relative particles are merely calculational devices. Hence, correlations, or the lack of them, between observables attached to the latter "particles" cannot be trusted in gauging entanglement.

Ex. 4: A single particle moving in $\mathbb{R}^{d}$. If there were any case where it makes no sense to talk about entanglement it would seem to be this one since it takes two to do the tango of entanglement. But do not be too hasty! Following quantum lore take the Hilbert space for the system to be $\mathcal{H}:=L_{\mathbb{C}}^{2}\left(\mathbb{R}^{d}\right)$ and the system algebra to be $\mathfrak{N}:=\mathfrak{B}\left(L_{\mathbb{C}}^{2}\left(\mathbb{R}^{d}\right)\right)$. This is not the end of the story, however. All infinite dimensional separable Hilbert spaces are isomorphic and, in particular, $L_{\mathbb{C}}^{2}\left(\mathbb{R}^{d}\right) \simeq L_{\mathbb{C}}^{2}\left(\mathbb{R}^{d}\right) \otimes L_{\mathbb{C}}^{2}\left(\mathbb{R}^{d}\right)$. Consequently, the system algebra is isomorphic to $\mathfrak{B}\left(L_{\mathbb{C}}^{2}\left(\mathbb{R}^{d}\right) \otimes L_{\mathbb{C}}^{2}\left(\mathbb{R}^{d}\right)\right) \simeq \mathfrak{B}\left(L_{\mathbb{C}}^{2}\left(\mathbb{R}^{d}\right)\right) \otimes$ $\mathfrak{B}\left(L_{\mathbb{C}}^{2}\left(\mathbb{R}^{d}\right)\right)$. The choice of a particular isomorphism between the system algebra $\mathfrak{B}\left(L_{\mathbb{C}}^{2}\left(\mathbb{R}^{d}\right)\right)$ and $\mathfrak{B}\left(L_{\mathbb{C}}^{2}\left(\mathbb{R}^{d}\right)\right) \otimes \mathfrak{B}\left(L_{\mathbb{C}}^{2}\left(\mathbb{R}^{d}\right)\right)$ specifies how the component algebras in the tensor product are to be identified as subalgebras of $\mathfrak{N}{ }^{39}$ In the present case there does not seem to be any choice of isomorphism that is more natural than any other. But no matter - let the choice be made by whatever means. We now have a tensor product decomposition of the system algebra into mutually commuting non-abelian subalgebras.

A sufficiently clever experimentalist will be able to specify a possible experimental setup to access the component subalgebra observables qua their identifications with system (one-particle) observables. Thus, if a system state 
fails to be a product state over the component subalgebras, the Pragmatist (if she is consistent) should declare a case of entanglement. The Realist will gleefully claim that this declaration is a reductio ad absurdum of the Pragmatic position; for (the Realist will claim) it is patently obvious that in the present example there are no genuine subsystems to be entangled, and the entanglement the Pragmatist claims to see is nothing other than the correlations between gerrymandered observables of an undivided unary system. Of course, the Realist concedes that new physics might change the situation, e.g. evidence might emerge indicating that the seemingly humble spinless particle is not structureless but is composed of subparticles over which real entanglement can take place. But failing such a discovery, the Realist sees no reason to treat the present example as anything other than empty mathematical conjuring.

The Realist need not tie his account to a particle ontology, as the above examples might erroneously be taken to indicate. Indeed, the Realist should tailor his account to the ontology that is compatible with the best available physical theories. In QFT local quantum fields on spacetime arguably take over from particles the role of the basic ontological furniture. Cluing in to this field ontology, the algebraic formulation of relativistic QFT posits a net of von Neumann algebras $\mathfrak{N}(\mathcal{O})$ associated with open bounded regions $\mathcal{O}$ of Minkowski spacetime $\mathcal{M}$. These local algebras are the natural candidates for subsystem algebras, and in bottom-up fashion the quasi-local global algebra $\mathfrak{N}(\mathcal{M})$ is identified with the von Neumann algebra generated by the subsystem algebras $\mathfrak{N}(\mathcal{O})$ as $\mathcal{O}$ ranges over all open bounded regions. There is no imminent threat here of ambiguity of entanglement since there are no are natural candidates for alternative decompositions of the system algebra.

I confess that I vacillate between Pragmatism and Realism. I feel the tug of some of the considerations the Realist puts forward. But I am also sensitive to the Pragmatist's retort to the Realist: 'You may want to label as faux entanglement those cases of entanglement over subalgebras you regard as not corresponding to genuine subsystems. But suppose I can describe possible experimental arrangements that access the observables of these subalgebras and also show how entanglement between these subalgebras can be put to work in quantum computing and information processing tasks. That entanglement may not be real enough for you, but it is real enough for me!' I am equally sensitive to the Realist's retort: 'I admit that in the situations you describe you are on to something real. But that something should not be called entanglement because entanglement is correlations between the 
observables of real subsystems.'

At this point the Realist-Pragmatist debate has ceased to be productive. But the debate has served its purpose in illustrating the possible reactions to the threat of rampant ambiguity of entanglement that results from the many different mathematically possible ways to decompose the system algebra into subalgebras. One can wonder how Schrödinger and the other pioneers of the new quantum theory would have situated themselves in the debate. In particular, how would Schrödinger's seminal 1935 paper on entanglement have been changed by an explicit recognition of the issues that underlie the debate?

\section{Entanglement for Indistinguishable Subsystems}

There is a sizable physics literature on entanglement for indistinguishable particles, containing different proposals for how to quantify entanglement for bosons and fermions. From the point of view on entanglement taken above it is hard to understand how to make sense of the debate over which proposal is correct. ${ }^{40} \mathrm{I}$ begin with a quick primer on indistinguishability in quantum physics.

\subsection{Indistinguishability}

I follow the usage that takes "identical (sub)systems" to mean (sub)systems with the same intrinsic or state-independent properties, such as mass. Thus, identical systems need not be indistinguishable. Further, the subsystems may or may not be plausibly described as particles, but I will follow the practice in referring to them as such. It suffices to illustrate the issues that arise from indistinguishability in terms of two-particle systems. The system Hilbert space is $\mathcal{H}=\mathcal{H}_{1} \otimes \mathcal{H}_{2}$, where $\mathcal{H}_{1}$ and $\mathcal{H}_{2}$ are the one-particle Hilbert spaces and, since the particles are supposed to be identical, $\mathcal{H}_{1} \simeq \mathcal{H}_{2} \simeq \mathfrak{h}$. The system algebra for the two identical particle system is $\mathfrak{N}_{i d}:=\Theta_{i d}^{\prime \prime}$, where $\Theta$ is the set of all self-adjoint operators on $\mathfrak{h} \otimes \mathfrak{h}$ that correspond to genuine observables. When $\Theta$ contains all self-adjoint operators $\mathfrak{N}_{i d}=\mathfrak{B}(\mathfrak{h}) \otimes \mathfrak{B}(\mathfrak{h})$.

To say that the particles are quantum mechanically indistinguishable is to say that are subject to permutation invariance. In the algebraic approach we will want to say what this invariance comes to in terms of the structure of the algebra of observables. In the widely accepted Messiah and Greenberg (1964) treatment of permutation invariance, the composite system von 
Neumann algebra of observables $\mathfrak{N}_{\text {indist }}:=\Theta_{\text {indist }}^{\prime \prime}$ is derived not by taking $\Theta_{\text {indist }}$ to be the set of all self-adjoint operators acting on $\mathfrak{h} \otimes \mathfrak{h}$ but rather to be the subset of self-adjoint operators that commute with the natural unitary representation of the permutation group $\mathfrak{s}_{2}$. The result is a superselection rule: the observable algebra $\mathfrak{N}_{\text {indist }}$ is now the proper subalgebra $\mathfrak{B}\left(\mathcal{H}_{\mathcal{S}}\right) \oplus \mathfrak{B}\left(\mathcal{H}_{\mathcal{A}}\right)$ of $\mathfrak{B}(\mathfrak{h}) \otimes \mathfrak{B}(\mathfrak{h})$ where $\mathcal{H}_{\mathcal{S}}$ and $\mathcal{H}_{\mathcal{A}}$ are respectively the subspaces of $\mathfrak{h} \otimes \mathfrak{h}$ spanned by symmetrized and antisymmetrized vectors. In the direct sum decomposition induced by imposing permutation invariance there are two types of indistinguishable particles, bosons described by the symmetric sector $\left(\mathcal{H}_{\mathcal{S}}, \mathfrak{B}\left(\mathcal{H}_{\mathcal{S}}\right)\right)$ and fermions described by the antisymmetric sector $\left(\mathcal{H}_{\mathcal{A}}, \mathfrak{B}\left(\mathcal{H}_{\mathcal{A}}\right)\right)$. Note that at this level of analysis there is no spinstatistics connection, so it is not a misnomer to speak of spinless fermions. The composite system algebra is now a Type I non-factor, and as a result the coincidence of vector states and pure states, holding in ordinary QM sans superselection rules, fails; e.g. a vector state that corresponds to a vector that superposes over different selection sectors is a mixed state. ${ }^{41}$

For three or more particles there is an additional superselection sector for "paraparticles"; the algebra for this sector is not a Type I factor. While this complication is important for understanding quantum statistics, it can be ignored for present purposes, and the discussion will continue in terms of the two particle case. ${ }^{42}$

\subsection{Measures of Entanglement for Indistinguishable Particles}

The physics literature contains attempts to provide a criterion for when and to what degree vector states encode entanglement for indistinguishable particles. I will illustrate for a two-fermion system one widely discussed criterion, which piggybacks on the Schmidt decomposition for distinguishable particles.

Recall from Section 8 the Schmidt bi-orthogonal decomposition for $|\Psi\rangle \in$ $\mathcal{H}_{1} \otimes \mathcal{H}_{2}:|\Psi\rangle=\sum_{i=1}^{r} \lambda_{i}\left|x_{i}\right\rangle_{1} \otimes\left|y_{i}\right\rangle_{2}$, where $\lambda_{i}>0$ and the $\left|x_{i}\right\rangle_{1}$ and $\left|y_{j}\right\rangle_{2}$ are ON bases for $\mathcal{H}_{1}$ and $\mathcal{H}_{2}$ respectively. The number $r$ of terms in the sum is called the Schmidt rank. The Schmidt decomposition is unique up to choice relative phases, unless two (or more) of the $\lambda_{i} \mathrm{~s}$ are equal, in which case the corresponding $\left|x_{i}\right\rangle_{1} \otimes\left|y_{i}\right\rangle_{2}$ can be replaced by linear combinations of each other. For the most well known EPR states all the $\lambda_{i}$ in the Schmidt biorthogonal decomposition are the same, but whether or not this is essential to the EPR set up is arguable. ${ }^{43}$ In any case, non-uniqueness of the decom- 
position does not affect the Schmidt rank $r$, which takes values from 1 to $\min \left\{\operatorname{dim}\left(\mathcal{H}_{1}\right), \operatorname{dim}\left(\mathcal{H}_{2}\right)\right\}$. If $r=1$ the state is a product state and there is no entanglement. For $r \geq 2$ the Schmidt rank can be used as a measure of the depth of entanglement.

The approach to entanglement of vector states for fermions to be described here is driven by the idea that since the anti-symmetric subspace $\mathcal{H}_{\mathcal{A}}$ of the fermion sector was formed by antisymmetrizing vectors from $\mathcal{H}=\mathcal{H}_{1} \otimes \mathcal{H}_{2}$, an analysis of entanglement for fermions must start by writing the state vector in an explicitly antisymmetric form (see, for example, Ghirardi and Marinatto 2004a, 2004b, 2005). Any vector $|\Phi\rangle \in \mathcal{H}_{\mathcal{A}}$ of a two fermion system can be written as

$$
|\Phi\rangle=\sum_{i, j} \Omega_{i j}\left|x_{i}\right\rangle_{1} \otimes\left|y_{j}\right\rangle_{2}
$$

where $\left|x_{i}\right\rangle_{1}$ and $\left|y_{j}\right\rangle_{2}$ are $\mathrm{ON}$ bases respectively for $\mathcal{H}_{1}$ and $\mathcal{H}_{2}$ and $\Omega_{i j}$ is an antisymmetric complex matrix satisfying the normalization condition $\operatorname{tr}\left(\Omega^{*} \Omega\right)=$ $-\frac{1}{2}$. In case $\operatorname{dim}\left(\mathcal{H}_{1}\right)=\operatorname{dim}\left(\mathcal{H}_{2}\right)=n$, the $\Omega_{i j}$ are the components of a complex $n \quad \mathrm{x} n$ matrix. For any such matrix there is a unitary $W$ such that $Z=W \Omega W^{T}$, where $Z$ is a block diagonal matrix

$$
Z=\operatorname{diag}\left[Z_{0} Z_{1} \ldots Z_{R}\right], \quad Z_{0}=0, Z_{i}=\left[\begin{array}{cc}
0 & z_{i} \\
-z_{i} & 0
\end{array}\right]
$$

with $z_{i}>0, i \in\{1,2, \ldots, R\}$. The $Z_{i}$ are called elementary Slater determinants. It follows that when $|\Phi\rangle$ is written using the new component bases $\left|x_{i}^{\prime}\right\rangle_{1}=\sum_{k} W_{i k}\left|x_{k}\right\rangle_{1}$ and $\left|y_{j}^{\prime}\right\rangle_{2}=\sum_{k} W_{i k}\left|y_{k}\right\rangle$ it becomes the sum of elementary Slater determinants:

$$
|\Phi\rangle=\sum_{i=1}^{R} z_{i} \frac{1}{\sqrt{2}}\left[\left|x_{i}^{\prime}\right\rangle_{1} \otimes\left|y_{i}^{\prime}\right\rangle_{2}-\left|y_{i}^{\prime}\right\rangle_{1} \otimes\left|x_{i}^{\prime}\right\rangle_{2}\right]
$$

The number $R$ of elementary Slater determinants in the sum is called the Slater rank. ${ }^{44}$

The Slater decomposition is analogized to the Schmidt decomposition for distinguishable particles, and the analogy is used to motivate using the Slater rank $R$ of a measure of the entanglement for fermions just as the Schmidt rank was used as a measure of entanglement for the distinguishable 
particles. Schmidt rank $r=1$ for distinguishable particles corresponds to no entanglement, so the argument from analogy says that Slater rank $R=$ 1 corresponds to no entanglement for fermions while Slater rank $R>1$ supposedly indicates entanglement for the fermions.

A similar analysis is applied to a boson state vector $|\Upsilon\rangle \in \mathcal{H}_{\mathcal{S}}$. Again, the idea is that since $\mathcal{H}_{\mathcal{S}}$ was originally formed by symmetrizing vectors from $\mathcal{H}=\mathcal{H}_{1} \otimes \mathcal{H}_{2}$, an analysis of entanglement for bosons must start from writing the state vector in an explicitly symmetric form. Thus, $|\Upsilon\rangle$ is written as

$$
|\Upsilon\rangle=\sum_{i, j} \Pi_{i j}\left|x_{i}\right\rangle_{1} \otimes\left|y_{j}\right\rangle_{2}
$$

where the $\Pi_{i j}$ are the components of an $n \times n$ symmetric matrix when $\operatorname{dim}\left(\mathcal{H}_{1}\right)=\operatorname{dim}\left(\mathcal{H}_{2}\right)=n$. Any such matrix can be diagonalized by a unitary transformation, resulting in the symmetric Schmidt form

$$
|\Upsilon\rangle=\sum_{i=1}^{R_{s}} b_{i}\left|x_{i}^{\prime}\right\rangle_{1} \otimes\left|x_{i}^{\prime}\right\rangle_{2}
$$

where the $b_{i}$ are positive real numbers. The number $R_{s}$ of terms on the sum gives the symmetric Schmidt rank. The argument at this juncture is more complicated than a simple appeal to analogy, but it concludes that a symmetric Schmidt rank of $R_{s} \geq 3$ is necessary as well as sufficient for entanglement of bosons.

\subsection{What are They Talking About?}

The mathematics described in the preceding subsection is intrinsically interesting, but one wonders what it has to do with entanglement as analyzed in the algebraic approach. The challenge can be posed by repeating once again the mantra of the algebraic approach: a system state is an expectation value functional on the system algebra, and entanglement of this state is entanglement with respect to a decomposition of the system algebra into subsystem algebras. Note that in some instances there may be no pertinent decomposition for the fermion sector $\left(\mathcal{H}_{\mathcal{S}}, \mathfrak{B}\left(\mathcal{H}_{\mathcal{S}}\right)\right)$ or for the boson sector $\left(\mathcal{H}_{\mathcal{A}}, \mathfrak{B}\left(\mathcal{H}_{\mathcal{A}}\right)\right)$. For example, if $\operatorname{dim}\left(\mathcal{H}_{\mathcal{S}}\right)$ is a prime number then (as noted above) there is no quantum entanglement ready tensor product decomposition for $\left(\mathcal{H}_{\mathcal{S}}, \mathfrak{B}\left(\mathcal{H}_{\mathcal{S}}\right)\right)$. But generally when there is one entanglement ready decomposition for one or both sectors then there are many. Which are the relevant decompositions for discussing entanglement? 
One response to the challenge would be to avoid it by refusing to specify the relevant decompositions and, thereby, acknowledging that the sense of entanglement being measured by symmetric Schmidt rank and Slater rank is not continuous with the sense of entanglement developed above for distinguishable particles. Much of the literature on entanglement of indistinguishable particles is written with an eye to applications to quantum information processing and quantum computing. The goal, sometimes explicit and sometimes implicit, is to identify and quantify the (so-called) entanglement of indistinguishable particles that is useful for information processing and computing. ${ }^{45}$ The claim on behalf of the Slater rank and the symmetric Schmidt rank measures, or alternative measures that have been proposed, would be that they do serve to identify situations useful for quantum information processing and computation, and they do so independently of the tensor product decompositions or more general ways of specifying physically relevant subalgebras of observables. I am skeptical about such claims since I don't understand how quantum computation and information processing can be analyzed without at least implicit reference to relevant subalgebras of observables and, thus without responding to the challenge. But if the claims do prove to be correct, I would still insist that Slater rank, symmetric Schmidt rank, etc. should be called measures of the usefulness for information processing, not a measure of entanglement. This is partly a terminological matter, but getting the right terminology is important in avoiding confusions and pseudo-issues.

A non-evasive direct response to the challenge would take us back to the Realist vs. Pragmatist debate initially posed in terms of distinguishable particles. In the present case the Realist might seem to have the upper hand since, pending the arrival of new physics (about sub-fermions and subbosons?), there does not seem to be any good physical basis on which to define decompositions of $\left(\mathcal{H}_{\mathcal{S}}, \mathfrak{B}\left(\mathcal{H}_{\mathcal{S}}\right)\right)$ and $\left(\mathcal{H}_{\mathcal{A}}, \mathfrak{B}\left(\mathcal{H}_{\mathcal{A}}\right)\right)$ corresponding to real subsystems and over which real entanglement can obtain. But for the Pragmatist the collapse of the distinction between real vs. virtual or fictitious subsystems is not surprising - it was always a suspect distinction. Nor is the collapse any impediment to discussing entanglement for indistinguishable particles since the same pragmatic factors that operate for distinguishable particles also operate in the present case. But the Realist and Pragmatist should agree on this much: however the decompositions of $\left(\mathcal{H}_{\mathcal{S}}, \mathfrak{B}\left(\mathcal{H}_{\mathcal{S}}\right)\right)$ and $\left(\mathcal{H}_{\mathcal{A}}, \mathfrak{B}\left(\mathcal{H}_{\mathcal{A}}\right)\right.$ are chosen, whether on Realist or Pragmatist grounds, the standard measures of entanglement can be applied to the chosen decomposition; 
there is no need to resort to Slater rank or the like.

Furthermore, the Realist and Pragmatist can find some neutral ground in a nearby setting; as described in the next subsection, the ground involves not indistinguishable particles per se but systems that employ indistinguishable particles.

11.4 State Entanglement for a System Employing Indistinguishable Particles

The following example is summarized from Zanardi (2002).

Ex. 5: Entanglement for fermionic lattices. Consider $N$ spinless fermions that may be localized at $L$ sites of a lattice. The single-particle Hilbert space is $\mathcal{H}_{L}:=\operatorname{span}\left\{\left|\psi_{l}\right\rangle\right\}$, where the $\left|\psi_{l}\right\rangle, l \in \mathbf{N}_{L}:=\{1, \ldots, L\}$, are the singleparticle wave functions describing localized states. The Hilbert space for the system is then the totally antisymmetric subspace $\mathcal{H}_{\mathcal{A}}(L, N)$ of $\mathcal{H}_{L}^{\otimes N}$. It is spanned by the ON vectors

$$
|A\rangle:=\frac{1}{\sqrt{N !}} \sum_{P \in \mathfrak{s}_{N}}(-1)^{|P|} \otimes_{l=1}^{N}\left|\psi_{j_{P(l)}}\right\rangle
$$

where $A:=\left\{j_{1}, \ldots, j_{N}\right\} \in \mathcal{P}_{L}^{N}$ the family of $N$-element subsets of $\mathbf{N}_{L}$.

In the abstract there is apparently no natural way to even get started on choosing a tensor product decomposition for $\mathcal{H}_{\mathcal{A}}(L, N)$. However, this intractable problem can be exchanged for a more tractable one by noting that an experimentalist interested in quantum information processing or computation is not concerned with the Hilbert space $\mathcal{H}_{\mathcal{A}}(L, N)$ but with another Hilbert space that throws away information about the details of the single particle wave functions; namely, the associated Fock space where the fermion number ranges from 0 to $L$, i.e. $\mathcal{H}_{\mathcal{A}}(L):=\oplus_{N=0}^{L} \mathcal{H}_{\mathcal{A}}(L, N)$. This space is $2^{L}$-dimensional and, thus, it admits a tensor product decomposition that is natural from the experimental point of view; namely, the $2^{L}$-dimensional $Q$ bit space $\left(\mathbb{C}^{2}\right)^{\otimes L}$ which has basis states $\left|m_{1}\right\rangle \otimes\left|m_{2}\right\rangle \otimes \ldots \otimes\left|m_{L}\right\rangle$, where the $m_{\mathrm{s}}$ take the values 0 or 1 with a 0 (respectively, 1 ) in the $k$ th place indicating that the $k$ th site is unoccupied (respectively, occupied). An isomorphism between $\mathcal{H}_{\mathcal{A}}(L)$ and $\left(\mathbb{C}^{2}\right)^{\otimes L}$ is given by

$$
\Lambda: \mathcal{H}_{\mathcal{A}}(L) \rightarrow\left(\mathbb{C}^{2}\right)^{\otimes L}:|A\rangle \rightarrow \otimes_{l=1}^{L}\left|\chi_{A}(l)\right\rangle
$$

where $\chi_{A}: \mathbf{N}_{L} \rightarrow\{0,1\}$ is the characteristic function of $A$. The implicit assumption here is that the experimentally accessible observables are not those 
belonging to $\mathfrak{B}\left(\mathcal{H}_{\mathcal{A}}(L, N)\right)$ but rather those belonging to $\mathfrak{B}\left(\mathcal{H}_{\mathcal{A}}(L)\right)$ which is isomorphic to the occupation number algebra $\mathfrak{B}\left(\mathbb{C}^{2}\right)^{\otimes L}$, a not unreasonable assumption for realistic experiments. ${ }^{46}$

The Pragmatist is certainly happy with this example since considerations of experimental accessibility play the leading role in determining the tensor product decomposition. And the Realist can be happy also since no claims are being made about entanglement of fermions per se, for which the Realist sees no principled basis. The entanglement, if there is such, is over the occupation number observables for the lattice sites, which by any measure count as real subsystems of the experimental system.

\section{Conclusions (and Diatribe)}

Whether or not the reader agrees with my claims on fine grained matters, I hope that I have made the case that there is still philosophical juice remaining in the much squeezed topic of quantum entanglement and that the algebraic formulation of quantum physics provides the appropriate tools to do the squeezing. The algebraic apparatus allows entanglement to be discussed for non-Type I factor algebras and thereby it provides for a uniform treatment of entanglement in ordinary QM and relativistic QFT, and it reveals a host of subtleties about entanglement that go unrecognized in treatments that ignore algebraic structure.

In the algebraic approach the discussion of entanglement naturally divides into two parts. The first part assumes that a choice of a decomposition of the system algebra into subsystem algebras has been made. Here a number of distinct but closely related criteria are available to discern whether or not there is genuine quantum entanglement over the chosen decomposition. That these criteria do not square with all intuitions is no embarrassment since there is rarely, if ever, a complete squaring between a formal explication and pre-analytic intuitions. Nor is multiplicity of distinct explications an embarrassment since they can seen as capturing different aspects of quantum entanglement. The second part of the discussion grapples with the problem of how to choose the decomposition into subsystem algebras. I framed the problem in terms of Realism vs. Pragmatism. There may be a better framing, but I picked the one I thought would resonate with the philosophical literature, hoping to engage the interest of philosophers who, for the most part, are seemingly oblivious to an issue on which they are well equipped 
to contribute. And I illustrated how the problem of choosing subsystem algebras is especially acute in defining entanglement for indistinguishable particles. Indeed, the proponents of various measures of entanglement for indistinguishable particles simply avoid the problem by failing to specify the relevant subsystem algebras, leading one to wonder whether they are talking about entanglement in some sense quite different from the standard ones developed for distinguishable particles.

One large issue I have touched on only tangentially is the relation between entanglement and the separability/independence of subsystems. Philosophers have much to say on these matters, some of it ill-advised and/or confused. ${ }^{47}$ I refer specifically to the line which, following Einstein's reassessment of the EPR paradox, holds that quantum entanglement somehow compromises the separability/independence (the "being thus" to use Einstein's phrase) of subsystems. ${ }^{48}$ On the contrary, separability/independence for subsystems is best discussed in terms of requirements on the subsystem algebras; and all such requirements that have been formulated in algebraic quantum theory, from mutual commutativity, to strict locality, the Schlieder property, $W^{*}$-independence, etc. through the split property, are satisfied in ordinary QM, which is the locus of much of the philosophical literature. And this is so even though deep quantum entanglement — as signaled by maximal violations of Bell's inequalities - is endemic among normal states. These separability/independence requirements have also been shown to hold in models of algebraic QFT for the algebras associated with relatively spacelike open and bounded regions of Minkowski spacetime, although again violations of the Bell inequalities are endemic. For algebras associated with unbounded spacetime regions higher-order separability/independence properties can fail, e.g. the algebras associated with relatively spacelike Rindler regions violate the split property. Here there may be something to the line that entanglement compromises separability/independence, but if so it is not due to entanglement per se but to the vicious nature of the entanglement - in the Rindler wedge example there are no normal product states. But surely this esoteric example is not what Einstein had in mind when he worried about separability.

Perhaps what Einstein, and those who follow his lead, have in mind when they think entanglement compromises separability/independence of subsystems is some sense of separability/independence that escapes the standard algebraic requirements. But apart from the trivial sense on which the very existence of quantum entangled states means ipso facto that the subsystems 
are not separable or independent, I have not seen any clear articulation of such a sense or why it would provide more illumination of the mysteries of quantum physics than saying: 'Here's one thing that is remarkable about quantum physics; namely, although subsystems of a composite quantum system can be separable/independent in every reasonable sense that can be formulated in terms of requirements on the subsystem algebras, states of the system can be entangled over the subsystems in a way that is impossible for classical systems. The mysteries of the quantum are then to be explored by understanding the nature of quantum entanglement, not by trying to blur the line between entanglement and separability/independence of physical systems. ${ }^{49}$ The difference between classical and quantum physics is not that subsystems cease to be separable/independent in moving from the former to the latter; the difference lies in the non-abelian character of the quantum observables, which goes hand-in-hand with the quantum nature of entanglement (Raggio's theorem). If you want to obsess about compromises to separability/independence ("being thus") of subsystems, worry about models of quantum phenomena where the subsystems show unmistakable signs of being joined at the hip because the subsystem algebras are not mutually commuting (such as violation of micro-causality in QFT) so that observables of the subsystems are not simultaneously measurable; or where the intersection of the subsystem algebras is non-trivial (violation of strict locality); or where normal states on the subsystem algebras cannot be extended to a joint normal state (violation of $W^{*}$-independence) so that subsystem states cannot be independently prepared; or where the system algebra is a not a tensor product of subsystem algebras, etc. But note that such nightmares do not haunt the actual world since successful quantum theories, whether set in classical or relativistic spacetime, do not display any of these non-separability/non-independence pathologies despite the fact that quantum entanglement of the deepest form is endemic among the normal states. ${ }^{\prime 50}$

I have a similar reaction to philosophical discussions of holism in quantum physics, where entanglement is often seen to promote holism. ${ }^{51}$ Once again I think that the holism/anti-holism distinction is best drawn in terms of requirements on algebras of observables. In relativistic QFT the axiom of strong additivity requires that the algebra $\mathfrak{N}\left(\cup_{j} \mathcal{O}_{j}\right)$ of observables associated with the union of any family of open bounded spacetime regions $\mathcal{O}_{j}$ coincides with the algebra $\vee_{j} \mathfrak{N}\left(\mathcal{O}_{j}\right)$ generated by the subalgebras $\mathfrak{N}\left(\mathcal{O}_{j}\right)$ associated with each of the individual regions - a precise way of capturing in algebraic 
terms the idea that the whole is not greater than the sum of its parts. If you want to worry about holism in QFT, worry about failures of Additivity. But note that such a worry doesn't arise because of entanglement since Additivity is compatible with quantum state entanglement in even the deepest form.

Of course, to be more than flag waving these remarks about separability and holism which go against the tide of philosophical commentary would need to be to be supported by detailed argumentation. But that is another project.

Acknowledgment I am grateful to an anonymous referee for suggestions that led to improvements of a earlier version of this article.

\section{Notes}

The final publication is available at link.springer.com; Erkenntnis DOI $10.1007 / \mathrm{s} 10670-014-9627-8$

1. With the rise of quantum computing and quantum information theory there has been a sea change in attitude towards entanglement: it is not something to be feared but rather is a resource to be exploited.

2. Only separable Hilbert spaces will be considered here.

3. For a classification of the types of von Neumann algebras, see Sunder (1986). An algebra $\mathfrak{N}$ is a factor just in case its center $\mathcal{Z}(\mathfrak{N}):=\mathfrak{N} \cap \mathfrak{N}^{\prime}=\mathbb{C} I$. Superselection rules involve algebras with non-trivial centers; see Section 10.

4. See Hamhalter (2003) for details.

5. There is also a more abstract version of the algebraic approach using $C^{*}$-algebras instead of von Neumann algebras. Each version has its own advantages. For the present topic the von Neumann algebra version seems the most illuminating.

6. Silently because texts on ordinary QM only rarely talk explicitly about algebras of observables. The case of indistinguishable particles will be treated in Section 11.

7. Product states are called "factorizable" in the philosophical literature, although this term is used specifically in the case where $\mathfrak{N}_{1} \vee \mathfrak{N}_{2}$ has a tensor product structure; see below.

8. See Bratteli and Robinson (1987, Corr. 2.3.21).

9. Let $(x, y, z, t)$ be an inertial coordinate system for Minkowski spacetime. Then the right Rindler wedge with vertex at the origin consists of those 
points $\infty>x>0, x^{2}-t^{2}>0$. Reflecting about the origin gives the left Rindler wedge.

10. This fact was apparently first noted by Araki; see Buchholz (1974, p. 292).

11. For an overview of these requirements, see Summers (1990, 2009).

12. To make sure that the tensor product algebra is a von Neumann algebra the overbar is added, i.e. $\mathfrak{N}_{1} \bar{\otimes} \mathfrak{N}_{2}:=\left(\mathfrak{N}_{1} \otimes \mathfrak{N}_{2}\right)^{\prime \prime}$. For Type I factors $\left(\mathfrak{N}_{1} \otimes \mathfrak{N}_{2}\right)^{\prime \prime}=\left(\mathfrak{N}_{1} \otimes \mathfrak{N}_{2}\right)$.

13. A double cone region is the interior of the intersection of the forward light cone of a spacetime point $p$ with the backward light cone of a point $q$ where $q$ lies to the chronological future of $p$.

14. See the following Section for substantiation of this last point.

15. In d'Espagnat's terminology, the mixture would then be "improper." For a discussion of the distinction between "proper" and "improper" mixtures see d'Espagnat (1971, Sec. 6.3).

16. Or, more cynically, one simply demands a criterion that will lend itself to formal proofs.

17. Much of the literature uses "separable" where I use "decomposable." The former term has become so loaded with (sometimes misleading) associations I prefer to use the latter.

18. The norm topology is the appropriate topology to use in taking limits since the set of normal states is closed in this topology. If non-normal states are considered then the appropriate topology is the $w^{*}$-topology. The set of all states, normal and non-normal, on a von Neumann algebra is the $w^{*}$ closure of convex linear combinations of pure states.

19. Note that, contrary to what formula (1) might suggest, decomposability does not require that in the density operator representation of the state the projectors in the decomposition must be orthogonal. The importance of this point will become apparent in Section 7 where the notion of "classicality" of states is introduced; this notion does require orthogonality.

20. See Bruß (2001) for a survey of various operational and non-operational criteria of entanglement.

21. Not quite so fast! What one would like is the result that (R1) is equivalent to

$\left(\mathrm{R} 2^{\prime}\right)$ no normal state on $\mathfrak{N}_{1} \vee \mathfrak{N}_{2}$ is (E1) entangled over $\mathfrak{N}_{1}$ and $\mathfrak{N}_{2}$.

$\left(\mathrm{R} 2^{\prime}\right)$ entails (R2) and, thus, (R1). But it is not obvious that (R1) entails $\left(\mathrm{R} 2^{\prime}\right)$ when $\mathfrak{N}_{1} \vee \mathfrak{N}_{2}$ is not $*$-isomorphic to $\mathfrak{N}_{1} \bar{\otimes} \mathfrak{N}_{2}$. 
22. So for any resolution of the identity $I_{\mathcal{H}_{1}}$ by a family $E_{j}$ of mutually orthogonal projectors in $\mathfrak{N}_{1}$, it must be the case that $\sum_{j} \mathcal{F}_{E_{j}}(\lambda)=1$ for all $\lambda \in \Lambda$; and similarly for $\mathcal{F}_{F}$.

23. The percentage of cases where the entanglement disappears can be upped to $66.6 \%$ but not above; see below.

24. Alternatively, reflecting on (3) may prompt revisiting the decision to develop a purely formal criterion of quantum entanglement that doesn't take into account the way in which a state is actually prepared.

25. What is being (implicitly) used here is the Lüders conditonalization rule for a non-selective projective measurement; see below.

26. A normal state $\omega$ on $\mathfrak{B}\left(\mathcal{H}_{1}\right) \otimes \mathfrak{B}\left(\mathcal{H}_{2}\right)$ is non-entangled iff its corresponding density operator can be written as $\rho^{1} \otimes \rho^{2}$. Taking spectral decompostions, $\rho^{1}=\sum_{i} \lambda_{i}^{1} E_{i}^{1}$ and $\rho^{2}=\sum_{j} \lambda_{j}^{2} E_{j}^{2}$, where the $E_{i}^{1}$ and $E_{j}^{2}$ are respectively one-dimensional projectors on $\mathcal{H}_{1}$ and $\mathcal{H}_{2}$ and $0 \leq \lambda_{i}^{1}, \lambda_{j}^{2} \leq 1$, it follows that $\rho^{1} \otimes \rho^{2}=\sum_{i, j} \lambda_{i}^{1} \lambda_{j}^{2} E_{i}^{1} \otimes E_{j}^{2}$, which is obviously classical. This argument comes from Li and Luo (2008).

27. Perhaps then it is proper to denote "non-classical" by $\left(\mathrm{E} \frac{1}{2}\right)$ to indicate that it lies between (E1) and (E0).

28. Needless to say, a selective measurement will generally result in a change of state, the post measurement state being either $\omega(E \bullet E) / \omega(\bullet)$ or $\omega((I-E) \bullet(I-E)) / \omega(I-E)$ depending upon whether the outcome is respectively Yes or No. Since it is not clear what, if any, information is gained in a non-selective measurement I have changed "allows acquisition of information" to "allows interrogation."

29. That $\omega$ is faithful to $\mathfrak{N}$ means that $\omega(A)=0 \Rightarrow A=0$ for all $A \in \mathfrak{N}$. If, as assumed, $\mathfrak{N}$ acts on a separable Hilbert space then it admits a faithful normal state. This is not necessarily so if $\mathfrak{N}$ acts on a non-separable $\mathcal{H}$.

30. And "vector state" can be substituted for "pure state" since in the case at hand the normal pure states coincide with the vector states.

31. Since the function $\beta(\omega)$ that determines a violation of the Bell inequalities is linear, for a mixed state $\omega=\sum_{j} \lambda_{j} \varphi_{j}, \beta(\omega)=\sum_{j} \lambda_{j} \beta\left(\varphi_{j}\right)$. So if each of the $\varphi_{j}$ gives a maximal violation of the Bell inequalities then so does the mixed state $\omega$.

32. The converse is not true. What is true is that if the restriction of a 
vector state on $\mathfrak{B}\left(\mathcal{H}_{1}\right) \otimes \mathfrak{B}\left(\mathcal{H}_{2}\right) \otimes \ldots \otimes \mathfrak{B}\left(\mathcal{H}_{N}\right)$ to any bi-partite subsystem algebra $\mathfrak{B}\left(\mathcal{H}_{m}\right) \otimes \mathfrak{B}\left(\mathcal{H}_{n}\right), m \neq n$, is not only a classical state but also takes the special form $\sum_{i} p_{i} E_{i}^{m} \otimes E_{j}^{n}$, where the $E_{i}^{m}$ and $E_{j}^{n}$ are minimal resolutions of the identities of $\mathcal{H}_{m}$ and $\mathcal{H}_{m}$ respectively, then the state vector of the $N$ partite state admits a generalized Schmidt decomposition.

33. Of course, how strong this result is will depend on what technical explication is given for "generic." The decoherence approach to resolving the measurement problem would presumably welcome (or even require?) a strong no-trickle down result. For an overview of decoherence and its consequences, see Schlosshauer (2007).

34. Does this present a problem for the decoherence program?

35. For the composite system Hilbert space Schrödinger used the space of $L_{\mathbb{C}}^{2}$ functions $\psi(x, y)$ where $x$ and $y$ are the coordinates of the two subsystems. Then the condition for a product state is that $\psi(x, y)$ is a product of a function of $x$ and a function of $y$.

36. In order to identify the component algebras in the tensor product algebra as subalgebras of the system algebra $\mathfrak{B}\left(L_{\mathbb{C}}^{2}\left(\mathbb{R}^{2 d}\right)\right)$ it is necessary to choose a particular isomorphism between the system algebra and the tensor product algebra. A natural choice is dictated if the position and momentum operators $Q$ and $P$ of the two-particle system are identified respectively with $q_{1} \otimes q_{2}$ and $p_{1} \otimes p_{2}$ where the $q$ 's and $p$ 's are the one-particle position and momentum operators. See also Ex. 4 below, where there is no natural choice of isomorphism.

37. Each of $\sigma_{1}^{x}, \sigma_{1}^{y}, \sigma_{1}^{z}$ commutes with each of $\sigma_{2}^{x}, \sigma_{2}^{y}, \sigma_{2}^{z}$. The other defining properties of the Pauli operators are that $\left(\sigma_{j}^{x}\right)^{2}=\left(\sigma_{j}^{y}\right)^{2}=\left(\sigma_{j}^{z}\right)^{2}=I$, and $\sigma_{j}^{x} \sigma_{j}^{y}=-\sigma_{j}^{y} \sigma_{j}^{x}=i \sigma_{j}^{z}, \sigma_{j}^{y} \sigma_{j}^{z}=-\sigma_{j}^{z} \sigma_{j}^{y}=i \sigma_{j}^{x}, \sigma_{j}^{z} \sigma_{j}^{x}=-\sigma_{j}^{x} \sigma_{j}^{z}=i \sigma_{j}^{y}$ for $j=1,2$.

38. Zanardi et al. (2004) use this example to motivate the slogan that "[E]ntanglement is always relative to a particular set of experimental capabilities" (p. 1; italics in the original); see also Zanardi (2001, 2002). It is unclear, however, whether they mean to fully endorse what I am calling Pragmatism.

39. If $\mathcal{I}: \mathfrak{N}_{1} \bar{\otimes} \mathfrak{N}_{2} \rightarrow \mathfrak{N}$ is a $*$-isomorphism, then $A \in \mathfrak{N}_{1}$ (respectively, $\left.B \in \mathfrak{R}_{2}\right)$ is identified with $\mathcal{I}(A \otimes I)$ (respectively, $\mathcal{I}(I \otimes B)$ ). Without such an identification the entanglement of a composite system state over the components of the tensor product algebra becomes inscrutable.

40. The reader interested in getting a sense of the recent physics literature 
on how to define and quantify entanglement for indistinguishable particles can start with the following sample: Amico et al. (2008), Bañuls et al. (2007), Dowling et al (2006), Eckert et al. (2002), Ghirardi et al. (2002), Ghirardi and Marinatto (2003, 2004a, 2004b, 2005), Giddings and Fisher (2002), Horodecki et al. (2009), Kaplan (2005), Li at al. (2001), Paškauskas and You (2001), Plastino et al. (2009), Schliemann et al. (2001), Shi (2003, 2004), Wang et al. (2006), Wiseman and Vaccaro (2003), Zanardi (2001, 2002), Zanardi and Wang (2002), and Zanardi et al. (2004).

41. It is sometimes said that superselection rules place limitations on the superposition principle. Strictly speaking this is false: a vector space is a vector space, is a vector space, ... , i.e. the linear combination of any two vectors belongs to the space. What is true that some linear combinations do not produce coherent superpositions, i.e. the linear combination is a vector that generates a mixed algebraic state. See Earman (2008).

42. The postulate of the commutativity of superselection rules asserts the commutativity of all unitary operators that commute with the set of $\Theta$ of all self-adjoint operators corresponding to genuine observables. This postulate fails for a system of three or more indistinguishable particles since the unitaries representing pair interchanges do not commute. For a discussion of these matters, see Earman (2008).

43. Without citing Schmidt (1906), Schrödinger (1935) states a version of the bi-orthogonal decomposition theorem, and he takes the equality of all of the $\lambda_{i}$ of the bi-orthogonal decomposition to be a necessary condition for the "Einstein-Podolsky-Rosen case" (p. 558).

44. Note that this procedure does not generalize beyond the two-fermion case, which should already arouse suspicion about whether it captures something fundamental.

45. Such entanglement is sometimes dubbed "accessible entanglement" (Wiseman and Vaccaro 2003) and sometimes "quantum entanglement" (Eckert at al. 2002) - which should not be confused with the sense of quantum entanglement discussed above.

46. The full story can be more complicated. For example, if the fermions are charged then charge superselection limits the genuine observables to a subalgebra $\mathfrak{B}\left(\mathcal{H}_{\mathcal{A}}(L)\right)$, namely the von Neumann algebra generated by all self-adjoint operators on $\mathcal{H}_{\mathcal{A}}(L)$ that commute with the number operator.

47. I realize that in saying this I am waving a red flag in front of the thundering herd of philosophers who pronounce on these matters. So be it. These bulls can only succeed in goring themselves more than they already 
have.

48. The EPR paradox is first presented in Einstein et al. (1935). A number of years later in "Quantum Mechanics and Reality" (1948) Einstein made it clear his real worry was what he perceived as the challenge that quantum physics poses to the "independent existence (the 'being-thus')" of (sub)systems. For attempts to clarify Einstein's worry, see Howard (1989) and Fine (1996).

49. Those who want to blur the line between separability/independence and entanglement end up not doing justice to either. An example of what I have in mind comes from Howard's (1989), which is concerned with a separability principle that is "a fundamental ontological principle governing the individuation of physical systems and their associated states" (p. 225). This principle requires that

(H) The joint state of the two systems is wholly determined by the separate states of the two component systems.

In a footnote Howard explains:

How the joint state is determined by the separate states depends on the details of a theory's mathematical formulation. At a minimum, the idea is that no information is contained in the joint state that is not already contained in the separate states ... (fn 2, p. 226)

None of the extant mathematical formulations of quantum physics gives a prescription for going from states on subsystems to a state on the composite system. But in the present context $(\mathrm{H})$ can be taken to require that for any pair of subsystem states there must be a unique extension to a joint stateotherwise there is a loss of information in passing from the composite system state to the component system states. This condition is indeed violated for entangled states. But this information loss criterion does not capture the difference between simple entanglement (E0) and genuine quantum entanglement ((E1), (E2), or (E3)) since information loss can occur for both types of states on $\mathfrak{N}_{1} \bar{\otimes} \mathfrak{N}_{2}$ when the subsystem algebras are non-abelian. When both subsystem algebras $\mathfrak{N}_{1}$ and $\mathfrak{N}_{2}$ are classical (= abelian) we know that there are no states (normal or non-normal) on $\mathfrak{N}_{1} \bar{\otimes} \mathfrak{N}_{2}$ that are (E1) entangled over $\mathfrak{N}_{1}$ and $\mathfrak{N}_{2}$. Nevertheless, even for abelian subsystem algebras there 
are normal entangled states on $\mathfrak{N}_{1} \bar{\otimes} \mathfrak{N}_{2}$ for which there is information loss in passing to the reduced subsystem states. But no one thinks that separability/independence of classical systems is threatened by such information loss.

Howard (1989) has been criticized by Fogel (2007) and Winsberg and Fine (2003) but on rather different grounds than the ones offered here.

50. One might also worry that in relativistic QFT the entanglement between relatively spacelike regions cannot be destroyed by local operations (see Clifton and Halvorson 2001). Here I have three comments. First, Valente (2013) argues that the Clifton-Halvorson no-go result rests on a overly restrictive notion of local operation. Second, the point at issue seems to me to relate less to the question of separability/independence of subsystems than to the question of how entrenched entanglement is in QFT. Third, if (contrary to what I am suggesting) there is a defensible no-go result here and if that result can be shown to compromise the "being thus" of subsystems, so be it. That is what would create a problem for separability/independence, not the existence or even ubiquity of entanglement per se.

51. For an authoritative overview, see Healey (2008).

\section{References}

Amico, L., Fazio, R., Osterloh, A., and Derdal, V. (2008). Entanglement in many-body systems. Reviews of Modern Physics, 80, 517-576.

Ancin, A, Andrianov, A, Costa, L., Jané, E., Latorre, J. I., and Tarrach, R. (2000). Generalized Schmidt decompositon and classification of threequantum-bit states. Physical Review Letters. 85, 1560-1563.

Bañuls, M.-C., Cirac, J. I., and Wolf, M. M. (2007). Entanglement in fermionic systems. Physical Review A, 76, 022311-1-13.

Bratteli, O. and Robinson, D. W. (1987). Operator algebras and quantum statistical mechanics 1, 2nd ed. New York:, Springer-Verlag.

Brauenstein, S. L., Mann, A., and Revzen, M. (1992). Maximal violations of Bell inequalities for mixed states. Physical Review Letters, 68, 3259-3261.

Bruß, D. (2002). Characterizing entanglement. Journal of Mathematical Physics, 43, 4237-4251.

Buchholz, D. (1974). Product states for local algebras. Communications in Mathematical Physics, 36, 287-304. 
Clifton R. and Halvorson H. (2001). Entanglement and open systems in algebraic quantum field theory. Studies in History and Philosophy of Modern Physics, 32, 1-31.

d'Espagnat, B. (1971). Conceptual foundations of quantum mechanics. Menlo Park, CA: W. A. Benjamin.

de la Torre, A. C., Goyeneche, D., and Leitao, L. (2010). Entanglement for all quantum states. European Journal of Physics, 31, 325-332.

Dowling, M. R., Doherty, A. C., and Wiseman, H. M. (2006). Entanglement of indistinguishable particles in condensed matter physics. Physical Review A, 73, 052323-1-12.

Earman, J. (2008). Superselection rules for philosophers. Erkenntnis, 69, $377-414$.

Eckert, K., Schliemann, J., Bruss, and Lewenstein, M. (2002). Quantum correlations in systems of indistinguishable particles. Annals of Physics, 299, 88-127.

Einstein, A. (1948). Quantum mechanics and reality. Dialectica, 2, 323-324.

Einstein, A., Podolsky, B. and Rosen, N. (1935). Can quantum-mechanical description of physical reality be considered complete? Physical Review, 47, $777-780$.

Fine, A. (1996). The shaky game: Einstein, realism, and the quantum theory, 2nd ed. Chicago: University of Chicago Press.

Fogel, B. (2007). Formalizing the separability condition on Bell's theorem. Studies in History and Philosophy of Modern Physics, 38, 920-937.

Garg, A. and Mermin, N. D. (1982). Correlation inequalities and hidden variables. Physical Review Letters, 49, 1220-1223.

Ghirardi, G., Marinatto, L., and Weber, T. (2002). Entanglement and properties of composite quantum systems: A conceptual and mathematical analysis. Journal of Statistical Physics, 108, 49-122.

Ghirardi, G. and Marinatto, L. (2003). Entanglement and properties. Fortschritte der Physik, 51, 379-387.

Ghirardi, G. and Marinatto, L. (2004a). Criteria for the entanglement of composite systems with identical particles. Fortschritte der Physik, 52, 10451051.

Ghirardi, G. and Marinatto, L. (2004b). General criteria for the entanglement of two indistinguishable particles. Physical Review A, 70, 102109-1-10. 
Ghirardi, G. and Marinatto, L. (2005). Identical particles and entanglement. Optics and Spectroscopy, 99, 386-390.

Giddings, J. R. and Fisher, A. J. (2002). Describing mixed spin-space entanglement of pure states of indistinguishable particles using occupation-number basis. Physical Review A, 66, 032305-1-11.

Gisin, N. (1991). Bell's inequality holds for all non-product states. Physics Letters A,154, 201-202.

Gisin, N. and Peres, A. (1992). Maximal violation of Bell's inequality for arbitrarily large spin. Physics Letters A, 162, 15-17.

Haag, R. (1992) Local quantum physics. Berlin: Springer Verlag.

Halvorson, H. and Clifton, R. (2000). Generic Bell correlation between arbitrary local algebras in quantum field theory. Journal of Mathematical Physics, 41, 1711-1718.

Hamhalter, J. (2003). Quantum measure theory. Dordrecht: Kluwer Academic.

Harshman, N. L. and Wickramasekara, S. (2007).Tensor product structures, entanglement, and particle scattering. Open Systems and Information Dynamics, 14, 341-351.

Healey, R. (2008). Holism and nonseparability in physics. In Stanford Encyclopedia of Philosophy. URL: http://plato.stanford.edu/entries/physicsholism/

Horodecki, R., Horodecki, P., Horodecki, M., Horodecki, K. 2009. Quantum entanglement. Reviews of Modern Physics, 81, 865-942.

Horuzhy, S. S. (1990). Introduction to algebraic quantum field theory. Dordrecht: Kluwer Academic.

Howard, D. (1989). Holism, separability, and the metaphysical implications of the Bell Experiments. In J. Cushing and E. McMullin (Eds.), Philosophical consequences of quantum theory: Reflections on Bell's theorem (pp. 224-253). Notre Dame, IN: University of Nature Dame Press.

Kaplan, T. A. (2005). On fermion entanglement. Fluctuation and Noise Letters, 5, C15-C22.

Li, N. and Luo, S. (2008). Classical states versus separable states. Physical Review A, 78, 024303-1-4.

Li, Y. S., Zeng, B., Liu, X. S., and Long, G. L. (2001). Entanglement in a two-identical-particle system. Physical Review A, 64, 054302-1-4. 
Luo, S. 2008. Using measurement-induced disturbance to characterize correlations as classical or quantum. Physical Review A, 7r, 022301-1-5.

Messiah, A. M. and Greenberg, O. W. (1964). Symmetrization postulate and its experimental foundation. Physical Review, 136, 248-267.

Modi, K., Paterek, T., Son, W., Vederal, V., and Williamson, M. (2010). Unified view of quantum and classical correlations. Physical Review Letters, 104, 080501-1-4.

Ollivier, H. and Zurek, H. (2002). Quantum discord: A measure of quantumness of correlations. Physical Review Letters, 88, 017901-1-4.

Paškauskas, R. and You, L. (2001). Quantum correlation in two-boson wave functions. Physical Review A, 64, 042310-1-4.

Peres, A. (1995). Higher order Schmidt decompositions. Physics Letters A, 202, 16-17.

Plastino, A. R., Manzano, D., Dehesa, J. S. (2005). Separability criteria and entanglement measures for pure states of $\mathrm{N}$ identical particles. European Physics Letters, 86, 2005-p1-2005-p5.

Popescu, S. (1994). Bell's Inequalities versus teleportation: What is nonlocality? Physical Review Letters, 72, 797-799.

Raggio, G. A. (1988). A remark on Bell's inequality and decomposable normal states. Letters in Mathematical Physics, 15, 27-29.

Schliemann, J., Cirac, I. I., Kuś, M., Lewenstein, M., and Loss, D. (2001). Quantum correlations in two-fermion systems. Physical Review A, 64, 0223031-9.

Schlosshauer, M. (2007). Decoherence and the quantum-to-classical transition. Berlin: Springer-Verlag.

Schmidt, E. (1906). Zur theorie linearen und nichtlinearen Integralgleichungen. Mathmatische Annalen, 63, 433-476.

Schrödinger, E. (1935). Discussion of probability relations between separated systems. Proceedings of the Cambridge Philosophical Society, 31, 555-563; 32 (1936), 446-451.

Shi, Y. 2003. Quantum entanglement of identical particles. Physical Review A, 67, 024301-1-4.

Shi, Y. (2004). Quantum entanglement in second-quantized condensed matter physics. Journal of Physics A, 37, 6807-6822. 
Summers, S. J. (1990). On the independence of local algebras in quantum field theory. Reviews in Mathematical Physics, 2, 201-247.

Summers, S. J. (2009). Subsystems and independence in relativistic microscopic physics. Studies in History and Philosophy of Modern Physics, 40, 133-141.

Sunder, V. (1986). An invitation to von Neumann algebras. Berlin: SpringerVerlag.

Tommasini, P. and Timmermans, E. (1998). The hydrogen atom as an entangled electron-positron pair. American Journal of Physics, 66, 881-886.

Valente, G. (2013). Local disentanglement in relativistic quantum field theory. Pre-print.

Wang, X.-H., Fei, S.-M., and Wu, K. 2006). Separability and entanglement of identical bosonic systems. Journal of Physics A, 39, L555-557.

Werner, W. F. 1989. Quantum states with Einstein-Podolsky-Rosen correlations admitting a hidden variable model. Physical Review A, 40, 4277-4281.

Winsberg, E. and Fine, A. (2003). Quantum life: interaction, entanglement, and separation. Journal of Philosophy, C, 80-97.

Wiseman, H. M. and Vaccaro, J. A. (2003). Entanglement of indistinguishable particles shared between two parties. Physical Review Letters, 91, 097902-1-4.

Zanardi, P. 2001. Virtual quantum systems. Physical Review Letters, 8\%, 077901-1-4.

Zanardi, P. 2002. Quantum entanglement for fermionic states. Physical Review A, 65, 042101-1-5.

Zanardi, P., Lidar, D. A., and Lloyd, S. 2004. quantum tensor product structures are observable induced. Physical Review Letters, 92, 06042-1-4.

Zanardi, P. and Wang, X. 2002. Fermionic entanglement in itererant systems. Journal of Physics A, 35, 7947-7959. 